\title{
Radial Gradients in Dust-to-gas Ratio Lead to Preferred Region for Giant Planet Formation
}

\author{
Yayaati Chachan ${ }^{1}$ (10), Eve J. Lee ${ }^{2,3}$ (i), and Heather A. Knutson ${ }^{1}$ (1) \\ ${ }^{1}$ Division of Geological and Planetary Sciences, California Institute of Technology, 1200 E. California Blvd., Pasadena, CA 91125, USA; ychachan@ caltech.edu \\ ${ }^{2}$ Department of Physics and McGill Space Institute, McGill University, 3550 rue University, Montréal, QC, H3A 2T8, Canada \\ ${ }^{3}$ Institute for Research on Exoplanets, Montréal, QC, Canada \\ Received 2020 December 20; revised 2021 June 2; accepted 2021 June 14; published 2021 September 24
}

\begin{abstract}
The Rosseland mean opacity of dust in protoplanetary disks is often calculated assuming the interstellar medium (ISM) size distribution and a constant dust-to-gas ratio. However, the dust size distribution and dust-to-gas ratio in protoplanetary disks are distinct from those of the ISM. Here we use simple dust evolution models that incorporate grain growth and transport to calculate the time evolution of the mean opacity of dust grains as a function of distance from the star. Dust dynamics and size distribution are sensitive to the assumed value of the turbulence strength $\alpha_{\mathrm{t}}$ and the velocity at which grains fragment $v_{\text {frag. }}$. For moderate-to-low turbulence strengths of $\alpha_{\mathrm{t}} \lesssim 10^{-3}$ and substantial differences in $v_{\text {frag }}$ for icy and ice-free grains, we find a spatially nonuniform dust-to-gas ratio and grain size distribution that deviate significantly from the ISM values, in agreement with previous studies. The effect of a nonuniform dust-to-gas ratio on the Rosseland mean opacity dominates over that of the size distribution. This spatially varying - that is, non-monotonic - dust-to-gas ratio creates a region in the protoplanetary disk that is optimal for producing hydrogen-rich planets, potentially explaining the apparent peak in the gas-giant planet occurrence rate at intermediate distances. The enhanced dust-to-gas ratio within the ice line also suppresses gas accretion rates onto sub-Neptune cores, thus stifling their tendency to undergo runaway gas accretion within disk lifetimes. Finally, our work corroborates the idea that low-mass cores with large primordial gaseous envelopes ("super-puffs") originate beyond the ice line.
\end{abstract}

Unified Astronomy Thesaurus concepts: Planet formation (1241); Protoplanetary disks (1300); Gas-to-dust ratio (638); Extrasolar gaseous giant planets (509); Mini Neptunes (1063)

\section{Introduction}

Dust opacity plays an important role in setting the temperatures and vertical structures of protoplanetary disks (e.g., Chiang \& Goldreich 1997; D’Alessio et al. 1998) and determines how rapidly a planet accretes its gaseous envelope (e.g., Stevenson 1982; Pollack et al. 1996; Ikoma et al. 2000). The temperature structure of the disk determines where various molecules can condense, resulting in a spatially and temporally varying division of elements between solid and gas phases (e.g., Hayashi 1981; Oberg et al. 2011). In the core accretion framework, dust opacity regulates the cooling of the envelope accreted by a growing planet (e.g., Lee et al. 2014; Piso \& Youdin 2014; Piso et al. 2015). Because the envelope accretion rate is cooling-limited during the hydrostatic phase of planetary growth, this dust opacity also has a strong influence on the final envelope mass.

In particular, cooling-limited accretion determines which planetary cores reach the threshold for runaway gas accretion within the gas disk lifetime and hence influences the giant planet occurrence rate. Radial velocity surveys indicate that giant planets inside 7 au only occur around 10\% of FGK stars, and they predominantly orbit their host stars at intermediate distances (3-5 au); their occurrence rate declines at both smaller and larger orbital distances (Cumming et al. 2008; Howard et al. 2012; Wittenmyer et al. 2016; Fernandes et al. 2019; Wittenmyer et al. 2020; Fulton et al. 2021; Rosenthal et al. 2021; complemented by direct imaging surveys, e.g., Bowler \& Nielsen 2018; Baron et al. 2019). It is unclear why giant planets preferably occur at intermediate distances. The water-ice line is typically assumed to play a role in making this region favorable for giant planet formation, primarily by facilitating the formation of massive cores (e.g., Morbidelli et al. 2015). However, the role of gas accretion in shaping the occurrence rate of giant planets remains largely unexplored.

Sub-Neptunes dominate the observed population of exoplanets with orbital periods less than 300 days (e.g., Batalha et al. 2013; Fressin et al. 2013; Morton \& Swift 2014; Dressing \& Charbonneau 2015; Petigura et al. 2018). The measured radii and masses of sub-Neptunes are consistent with hydrogen and helium envelope mass fractions of a few percent (Wolfgang \& Lopez 2015; Ning et al. 2018), despite the fact that some of these planets have cores massive enough $\left(\gtrsim 10 M_{\oplus}\right)$ to reach the threshold for runaway gas accretion. What regulates the envelope mass fraction at a few percent? It has been suggested that the accretion of material with high dust opacity could prevent these planets from amassing significantly larger envelopes (e.g., Lee et al. 2014; Chen et al. 2020). Here we revisit this idea and explore why sub-Neptunes might be expected to form close in, whereas gas giants are more common at larger orbital separations.

Determining the dust opacity at a given location in the protoplanetary disk is a nontrivial task, as it depends on the poorly known optical properties (composition and structure), size distribution, and dust-to-gas ratio, all three of which are intricately coupled to the protoplanetary disk's structure and evolution. Previous studies in both the protoplanetary disk and planet formation literature (e.g., Alexander \& Ferguson 1994a; Bell \& Lin 1994) have generally elected to adopt a single global value for the dust-to-gas ratio and a power-law size distribution (both the power-law index and the bounding grain sizes) that is akin to that of dust in the interstellar medium 
(ISM). However, such a prescription is too simplistic to calculate the mean opacity due to dust grains in a protoplanetary disk. Dust grains in protoplanetary disks grow to sizes that are significantly larger (millimeter-centimeter size; e.g., Miyake \& Nakagawa 1993; Testi et al. 2003; Draine 2006; Andrews 2015) than the largest submicron-sized grains in the ISM (Draine \& Lee 1984). A larger maximum grain size redistributes dust mass from smaller grains to larger grains, which significantly alters the short-wavelength and mean opacities of protoplanetary disks (e.g., D'Alessio et al. 2001).

Fortunately, advances in our understanding of grain coagulation and the role of fragmentation and radial drift in limiting grain growth now make it possible to calculate the grain size distribution as a function of location in protoplanetary disks (Brauer et al. 2008; Birnstiel et al. 2010, 2011). In a recent study, Savvidou et al. (2020) assessed the effect of varying grain size distribution from coagulation and fragmentation on the Rosseland mean opacity and the thermal structure of the disk but without taking dust transport into account. Transport of dust due to radial drift, gas drag, and turbulent diffusion leads to a radially varying dust-to-gas ratio, which may significantly alter dust opacity. This, in turn, leads to a location dependence of the gas accretion rates onto planetary cores. The gas accretion rate depends on the envelope's ability to cool at the innermost radiative-convective boundary (RCB). For envelopes in which dust opacity dominates, the sublimation of dust leads to the formation of an intermediate radiative zone, and the innermost $\mathrm{RCB}$ is set by the $\mathrm{H}_{2}$ dissociation front (Lee et al. 2014). Cooling at the innermost RCB is then controlled by $\mathrm{H}^{-}$opacity, which depends on the availability of free electrons provided by metals. These metals are mostly present in the dust initially, so the radially varying dust-to-gas ratio of the accreted material affects the rate at which the envelope can cool.

In this work, we use a published dust evolution model to calculate the spatial and temporal evolution of the dust-to-gas ratio in a protoplanetary disk (Birnstiel et al. 2012) in Section 2. We then calculate the corresponding Rosseland mean opacity using an approximate size distribution scheme to determine the grain size distribution as a function of distance from the star (Birnstiel et al. 2015). In Section 3, we compute the disk opacity from dust evolution models as a function of radial distance, height from midplane, and time and show that our results differ starkly from the usual ISM opacity values. We then use our updated opacity values to calculate gas accretion rates onto planetary cores using the analytical scaling laws from Lee \& Chiang (2015) and discuss the consequences of our work for the formation of gas giants, sub-Neptunes, and "super-puffs" (low-mass planets with sizes beyond $\sim 4 R_{\oplus}$ ) in Section 4. We summarize our results and suggest potential directions for future work in Section 5.

\section{Models}

\subsection{ISM Size Distribution}

The ISM size distribution is usually described using a power-law distribution,

$$
n(a)=A a^{\beta},
$$

where $n$ is the number of particles per unit volume per unit size interval, $A$ is a normalization factor that depends on the assumed dust-to-gas ratio and minimum and maximum grain sizes, and $\beta$ is the power-law index that characterizes how bottom- or top-heavy the size distribution is. The power-law index $\beta$ and minimum and maximum grain sizes $\left(a_{\min }\right.$ and $\left.a_{\text {max }}\right)$ are typically chosen to be -3.5 and 0.005 and $0.25 \mu \mathrm{m}$, respectively, which fit the observed extinction law in the diffuse ISM (Mathis et al. 1977; Laor \& Draine 1993; Alexander \& Ferguson 1994b). Although there are small variations in the values used for these parameters in the published literature, especially $a_{\min }$ and $a_{\max }$, they do not make an appreciable difference for the calculated opacity. The value for the normalizing constant $A$ is given by

$$
A=\frac{3 \rho_{d}(\beta+4)}{4 \pi \rho_{s}\left(a_{\max }^{\beta+4}-a_{\min }^{\beta+4}\right)},
$$

where $\rho_{s}=1.675 \mathrm{~g} \mathrm{~cm}^{-3}$ is the material density of the dust grain fixed to a value appropriate for the DSHARP mixture (see Section 2.3), $\rho_{d}$ is the density of dust in the disk, $\rho_{g}$ is the density of the disk gas, and $\epsilon=\rho_{d} / \rho_{g}$ is the dust-to-gas ratio. The ISM dust opacity is typically calculated assuming a global value of $\epsilon=0.01$ for the entire protoplanetary disk. For $\rho_{g}$, we use the gas density in the disk midplane obtained from our protoplanetary disk model, which we describe in the next section.

\subsection{Protoplanetary Disk Model}

We use the publicly available code twopoppy to model the structure of a protoplanetary disk and the dynamics of dust and gas. ${ }^{4}$ The methods and algorithms used in twopoppy are described in Birnstiel et al. (2012), and we will present a brief overview here for completeness. We consider a protoplanetary disk of mass $0.1 M_{*}$ around a protostar of mass $M_{*}=0.7 M_{\odot}$. The stellar effective temperature $\left(T_{*}\right)$ and radius $\left(R_{*}\right)$ are set to $4010 \mathrm{~K}$ and $1.806 R_{\odot}$, respectively. We assume that the disk is passively heated, and its temperature structure therefore takes the following form (e.g., Chiang \& Goldreich 1997; D’Alessio et al. 1998):

$$
T(r)=\left[\phi T_{*}^{4}\left(\frac{R_{*}}{r}\right)^{2}+T_{0}^{4}\right]^{1 / 4},
$$

where $r$ is the cylindrical distance from the star, $T_{0}=7 \mathrm{~K}$ is a constant, and $\phi=0.05$ is the angle between the incident radiation and disk surface ("flaring" angle). The sound speed $c_{s}$ is defined as $\sqrt{k_{\mathrm{B}} T / \mu m_{\mathrm{H}}}$, where $k_{\mathrm{B}}$ is the Boltzmann constant, $\mu=2.3$ is the mean molecular weight of the gas, and $m_{\mathrm{H}}$ is the mass of the hydrogen atom. Our neglect of heating due to viscous dissipation leads to an underestimation of the temperature in the inner regions of the disk but greatly simplifies the determination of the temperature structure. This choice does not have a significant effect on the position of the water-ice line (see Section 3.6 for a brief discussion). We note that accounting for the varying opacities that arise from the growth and transport of grains into the temperature profile is outside the scope of this paper (see, e.g., Savvidou et al. 2020, for recent attempts in this direction).

\footnotetext{
4 The original public repository is available at https://github.com/birnstiel/twopop-py. A fork of this repository with the changes implemented in our work is available at https://github.com/y-chachan/two-pop-py/tree/rad_grad_d2g.
} 
The gas surface density $\left(\Sigma_{\mathrm{g}}\right)$ is evolved following the fluid equations of the viscously spreading accretion disk (LyndenBell \& Pringle 1974),

$$
\frac{\partial \Sigma_{\mathrm{g}}}{\partial t}=\frac{3}{r} \frac{\partial}{\partial r}\left[r^{1 / 2} \frac{\partial}{\partial r}\left(\nu \Sigma_{\mathrm{g}} r^{1 / 2}\right)\right],
$$

whose self-similar solution (at time zero) is used to set the initial surface density profile for our calculation,

$$
\Sigma_{\mathrm{g}}(r)=C\left(\frac{r}{r_{c}}\right)^{-p} \exp \left[-\left(\frac{r}{r_{\mathrm{c}}}\right)^{2-p}\right],
$$

where $C$ is a constant to be normalized by the assumed disk gas mass, $\nu$ is the kinematic viscosity with a power-law radial profile $\left(\nu=\nu_{c}\left(r / r_{c}\right)^{p}\right)$, and $r_{\mathrm{c}}$ is a characteristic radius of the disk. Following Birnstiel et al. (2012), we set $p=1$ and $r_{\mathrm{c}}=200 \mathrm{au}$ in our work. The viscosity $\nu=\alpha_{\mathrm{t}} c_{s} \mathrm{H}_{\mathrm{gas}}$ is parameterized using the Shakura-Sunyaev turbulence parameter $\alpha_{\mathrm{t}}$ (Shakura \& Sunyaev 1973), the sound speed $c_{s}$, and the gas scale height $H_{\text {gas }}=c_{s} / \Omega$, where $\Omega$ is the Keplerian frequency.

The initial dust surface density is set as $\epsilon$ times the initial gas surface density given in Equation (5), where $\epsilon=0.01$. The dust surface density evolution and dynamics of dust are modeled using just two representative grain sizes in the disk (hence the name twopoppy): the spatially and temporally constant monomer size $a_{0}$ and a large grain size $a_{1}$ that depends on time and location in the disk. We fix $a_{0}=0.005 \mu \mathrm{m}$ to align this variable with the minimum grain size in the ISM size distribution. These small grains rapidly coagulate to form agglomerates that are many orders of magnitude larger in size. Their growth is limited by processes such as turbulent fragmentation and radial drift. These limiting sizes are what set the value of $a_{1}$ as a function of time and $r$, and they are discussed in greater detail later in this section.

Splitting the dust population into two allows us to capture the qualitatively different dynamical behavior of large and small grains. Small grains are well coupled to the gas and unable to maintain large relative velocities with respect to the gas. On the other hand, large grains are slightly decoupled from the gas and respond to pressure gradients on relatively short timescales. The total surface dust density $\left(\Sigma_{\mathrm{d}}\right)$ is the sum of the surface density of small $\left(\Sigma_{0}\right)$ and large $\left(\Sigma_{1}\right)$ grains and can consequently be modeled using a single advection-diffusion equation:

$$
\frac{\partial \Sigma_{\mathrm{d}}}{\partial t}+\frac{1}{r} \frac{\partial}{\partial r}\left[r\left(\Sigma_{\mathrm{d}} \bar{u}-D_{\mathrm{gas}} \Sigma_{\mathrm{g}} \frac{\partial}{\partial r}\left(\frac{\Sigma_{\mathrm{d}}}{\Sigma_{\mathrm{g}}}\right)\right)\right]=0 .
$$

Here $\bar{u}$ is the mass-weighted radial velocity of dust grains and $D_{\text {gas }}$ is the gas diffusivity. A derivation of this equation is available in the appendix of Birnstiel et al. (2012).

The Stokes number $\mathrm{St}$ is the dust grain stopping time under gas aerodynamic drag in units of local orbital time. Dust grains smaller than the gas particle mean free path are in the Epstein drag regime, and their Stokes numbers follow

$$
\mathrm{St}=\frac{\pi}{2} \frac{a \rho_{s}}{\Sigma_{\mathrm{g}}} .
$$

Detailed dust growth and evolution simulations indicate that grains will continue to grow until they reach a size $(\mathrm{St} \sim 0.1-1)$ where fragmentation due to collisions and/or loss to radial drift become significant (e.g., Brauer et al. 2008; Birnstiel et al. 2010). For grains in this size range, velocity differences between grains due to turbulence become larger $(\Delta u \propto \sqrt{\mathrm{St}}$; Ormel \& Cuzzi 2007), and collisions are more likely to lead to fragmentation instead of growth. This limits the maximum Stokes number and corresponding size $a_{\text {frag }}$ that the grains can reach,

$$
\begin{gathered}
\mathrm{St}_{\text {frag }}=\frac{1}{3 \alpha_{\mathrm{t}}} \frac{v_{\mathrm{frag}}^{2}}{c_{s}^{2}}, \\
a_{\text {frag }}=\frac{2}{3 \pi} \frac{\sum_{\mathrm{g}}}{\rho_{s} \alpha_{\mathrm{t}}} \frac{v_{\text {frag }}^{2}}{c_{s}^{2}},
\end{gathered}
$$

where $v_{\text {frag }}$ is the fragmentation velocity of dust grains.

The rate of radial drift is maximized for particles marginally coupled to gas (St 1; Weidenschilling 1977; Chiang \& Youdin 2010),

$$
u_{\text {drift }}=-\frac{2 u_{\eta}}{\mathrm{St}+\mathrm{St}^{-1}},
$$

where $u_{\eta}=-\gamma c_{s}^{2} / 2 v_{K}$ is the drift velocity, $v_{\mathrm{K}}$ is the Keplerian velocity, and $\gamma=|d \ln P / d \ln r|$ is the power-law index characterizing the dependence of pressure on distance from the star. In some regions of the disk, particles may drift radially faster than they can grow to the size at which fragmentation dominates. In these regions, the radial drift sets an upper limit on the particle size $a_{\text {drift }}$ :

$$
a_{\mathrm{drift}}=\frac{2}{\pi} \frac{\Sigma_{\mathrm{d}}}{\rho_{s} \gamma} \frac{v_{K}^{2}}{c_{s}^{2}}
$$

At early times in the disk evolution, the particle growth rate can also be a limiting factor for grain growth and set the maximum particle size. This can be true even at late times in the outer disk where the growth timescales $\left(\tau_{\text {grow }} \simeq 1 / \epsilon \Omega\right)$ are longer. Relative velocities due to radial drift can also lead to fragmentation, but this effect is only relevant at early times for models with low turbulence $\left(\alpha_{\mathrm{t}}=10^{-4}\right)$. As the dust-to-gas ratio in such a region declines due to inward drift, the size limit set by radial drift becomes smaller than the one set by driftinduced fragmentation. In the two-population model for dust evolution, the large grain size $a_{1}$ is fixed to a fraction of the maximum grain size that is determined by calibrating the twopoppy model to the full simulations (Birnstiel et al. 2012). The maximum particle size limit therefore plays an important role in determining the dynamics of the large grains in the disk. Since most of the dust mass tends to be concentrated in the largest grains, which are also the most susceptible to radial drift, the dust-to-gas ratio of the disk can evolve significantly over time.

The turbulence parameter $\alpha_{\mathrm{t}}$ and fragmentation velocity $v_{\text {frag }}$ are two of the most important parameters for determining the maximum particle size. The classically quoted range of values for $\alpha_{\mathrm{t}}$ is $10^{-4}-10^{-2}$ (e.g., Turner et al. 2014). However, recent studies of line broadening and dust settling in protoplanetary disks suggest that $\alpha_{\mathrm{t}}$ is closer to the lower end of this range (Mulders \& Dominik 2012; Flaherty et al. 2015; Pinte et al. 2016; Flaherty et al. 2017, 2018). We therefore adopt $\alpha_{\mathrm{t}}=10^{-3}$ 
for our baseline model and comment on the consequences of varying $\alpha_{\mathrm{t}}$ in Section 3.3. ${ }^{5}$

Both theoretical studies and experiments have long suggested a significant difference between the fragmentation velocities of ice-free and icy dust (Poppe et al. 2000; Blum \& Wurm 2008; Wada et al. 2013; Gundlach \& Blum 2015). Most commonly, ice-free silicate dust is assumed to have a fragmentation velocity of $1 \mathrm{~m} \mathrm{~s}^{-1}$, while icy grains have a fragmentation velocity closer to $10 \mathrm{~m} \mathrm{~s}^{-1}$ (e.g., Birnstiel et al. 2010; Pinilla et al. 2016; Drcazkowska \& Alibert 2017). Such a difference in fragmentation velocity would lead to an abrupt change in the dust emission spectral index at the water-ice line (Banzatti et al. 2015), and there is observational evidence to support the occurrence of this phenomenon (Cieza et al. 2016). This increase in fragmentation velocity for dust exterior to the water-ice line has also been invoked to explain the architecture of the solar system and exoplanetary systems (e.g., Morbidelli et al. 2015; Venturini et al. 2020), as well as planetesimal formation (Drcazkowska \& Alibert 2017).

Despite this apparent consensus, recent theoretical and laboratory studies have begun to cast doubt on this story. Previous studies attributed the change in $v_{\text {frag }}$ to an order-ofmagnitude difference in the surface energies of icy and ice-free dust grains, but recent experimental work now suggests that their surface energies may in fact be quite similar (Gundlach et al. 2018; Steinpilz et al. 2019). Other studies conclude that the fragmentation velocity might exhibit a more complicated and non-monotonic dependence on temperature (e.g., Gundlach et al. 2018; Musiolik \& Wurm 2019), and this topic remains an area of active debate in the community (e.g., Kimura et al. 2020). In this study, we adopt the standard values of $1 \mathrm{~m} \mathrm{~s}^{-1}$ for ice-free and $10 \mathrm{~m} \mathrm{~s}^{-1}$ for icy grains for our baseline case, as these are close to the values derived from dynamical collision experiments. We assume that the ice line is located where the disk temperature drops below approximately $T=200 \mathrm{~K}$, which places the ice line at $0.75 \mathrm{au}$ in all of our models. We use Gaussian convolution to smoothly increase $v_{\text {frag }}$ from $1 \mathrm{~m} \mathrm{~s}^{-1}$ at $250 \mathrm{~K}$ to $10 \mathrm{~m} \mathrm{~s}^{-1}$ at $150 \mathrm{~K}$ (e.g., Birnstiel et al. 2010). In Section 3.3, we also present alternative models where we vary the value of $v_{\text {frag }}$ both within and beyond the ice line and show that our results are qualitatively similar for a significant part of the plausible parameter space.

We utilize the approximations from Birnstiel et al. (2015; Equations (6)-(8) in their paper) that are implemented in twopoppy to reconstruct the full grain size distribution in the protoplanetary disk, which we need in order to calculate the corresponding dust opacity. We also modified the twopoppy code to include the size limit set by drift-induced fragmentation in the size distribution calculation, which in the default version of the code is approximated by the radial drift-limited grain size instead (see Birnstiel et al. 2012 for a discussion of the validity of this approximation). These approximations match the detailed simulations reasonably well but can underestimate the number density of small grains. Although this will affect the opacity of the disk at short wavelengths (e.g., $\sim 1 \mu \mathrm{m}$ ), we find that it only has a modest effect on the Rosseland mean opacity. We quantify this effect by comparing the mean opacity from this approximate method to the more accurate coagulation-fragmentation models from Birnstiel et al. (2011) in the

\footnotetext{
5 We note that we use the same $\alpha_{t}$ for both the global disk gas evolution and the turbulent stirring of dust. In reality, these two can be different (see, e.g., Carrera et al. 2017; Drcazkowska \& Alibert 2017).
}

fragmentation-dominated region of the protoplanetary disk and find that the opacity from the approximate method is a factor of 2 smaller. In regions dominated by radial drift, a change in the assumed power-law index for the size distribution can also affect the number of small particles. However, since radial drift tends to dominate in the outer colder parts of the disk, the mean opacity in this region is dominated by slightly larger grains $(\sim 100 \mu \mathrm{m})$, which have a more robustly determined number density.

So far, we have discussed grain sizes, distributions, and opacities in the framework of a vertically integrated (2D) disk. If we wish to explore the 3D disk structure, we can extend these $2 \mathrm{D}$ models by using some reasonable approximations to calculate the density of dust and gas as a function of height from the midplane. This exercise is particularly valuable for planet formation models because growing protoplanets might not accrete most of their gas from the midplane (see Section 4). We assume a Gaussian vertical profile with a scale height $H_{\text {gas }}(r)=c_{s} / \Omega$ for the gas. The midplane gas density is then given by $\rho_{\mathrm{g}, 0}=\Sigma_{g} / \sqrt{2 \pi} H_{\mathrm{gas}}$ (Equation (7), which gives the expression for $\mathrm{St}$ in the midplane, also used this assumption). Dust sediments toward the midplane and is carried upward by turbulent diffusion, so its vertical density distribution is significantly different from that of the gas. We use the expression for the steady-state vertical distribution of dust derived by Fromang \& Nelson (2009),

$$
\rho_{\mathrm{d}}(z, a)=\rho_{\mathrm{d}, 0} \exp \left[-\frac{\mathrm{St}_{0}}{\alpha_{\mathrm{t}}}\left(\exp \left(\frac{z^{2}}{2 H_{\text {gas }}^{2}}\right)-1\right)-\frac{z^{2}}{2 H_{\text {gas }}^{2}}\right],
$$

where $\rho_{\mathrm{d}, 0}(a)$ is the dust density and $\operatorname{St}_{0}(a)$ is the Stokes number in the midplane for a particular grain size. In reality, the vertical scale height for dust should be set by either turbulent diffusion or the Kelvin-Helmholtz shear instability, depending on which is larger at a given disk location (Rosenthal \& Murray-Clay 2018). We find that for our fiducial model, turbulent diffusion sets the dust scale height throughout the disk. The Kelvin-Helmholtz shear instability only comes into play for models with low turbulence strength $\left(\alpha_{\mathrm{t}}=10^{-4}\right)$ at large distances (30-100 au) and early times (0.1-1 Myr). For this subset of models, incorporating its effect on the vertical dust distribution decreases the final gas-to-core mass ratio (GCR) for a $15 M_{\oplus}$ core by at most $15 \%$ if accretion stops at $1 \mathrm{Myr}$. Continued gas accretion beyond this time wipes out the effect of incorporating Kelvin-Helmholtz instability in our analysis. Since accounting for Kelvin-Helmholtz instability has a negligible impact on our results, we choose to omit it from our work.

\subsection{Calculation of Dust Opacity}

The composition of dust grains in protoplanetary disks is a topic of active research (see recent review by Oberg \& Bergin 2021). We adopt the grain composition prescribed in the DSHARP survey papers and use the publicly available tools generously provided by the survey team for the calculation of grain properties (Birnstiel et al. 2018). The DSHARP composition mixture consists of water ice (optical properties from Warren \& Brandt 2008), "astrosilicates" (Draine 2003), and refractory organics and troilite (FeS; Henning \& Stognienko 1996). The 
Bruggeman mixing rule is employed to obtain the optical constants for the mixture. We adopt the same grain composition for the entire disk, as removing water from our mixture has only a small effect $(\lesssim 15 \%$, accounting for the difference in grain densities and optical properties but keeping the grain size distribution fixed) on the calculated opacity. Our simulations also do not account for the effect of condensation/sublimation on grain size and mass for particles moving across the ice line when calculating the grain size distribution. For the adopted DSHARP mixture, water's sublimation would reduce the dust mass by only $20 \%$ within the ice line. Accounting for the reduced mass and increased density of ice-free grains would reduce the grain size by $\sim 15 \%$, which will have some effect on their dynamics. However, these effects are negligible compared to the other sources of uncertainty in our model.

We use Mie theory to calculate the dust opacity. Our Mie code is publicly available as part of PLATON (Zhang et al. 2019, 2020), which uses the algorithm outlined by Kitzmann \& Heng (2018). For particle sizes and wavelengths for which the full Mie treatment is impracticable, we resort to widely used approximations. We use the geometric optics limit to calculate the absorption cross section of particles for which $|m| x>1000$ and $|m-1| x>0.001$, where $m$ is the complex refractive index of the particle and $x=2 \pi a / \lambda$ is the size parameter (here $a$ is the particle size, and $\lambda$ is the wavelength; van de Hulst 1957). Specifically, we use the approximation described in Laor \& Draine (1993), which uses the extinction coefficient calculated using the Rayleigh-Gans approximation $\left(Q_{\mathrm{RG}}\right)$ to obtain the extinction coefficient in the geometric optics limit $\left(Q_{\text {ext }}\right)$,

$$
\begin{gathered}
Q_{\mathrm{ext}} \approx \frac{Q_{\mathrm{RG}}}{\left(1+0.25 Q_{\mathrm{RG}}^{2}\right)^{1 / 2}}, \\
Q_{\mathrm{RG}}=\frac{32|m-1|^{2} x^{4}}{27+16 x^{2}}+\frac{8}{3} \operatorname{Im}(m) x,
\end{gathered}
$$

where $\operatorname{Im}(m)$ is the imaginary part of the refractive index.

Once we have calculated the absorption coefficient for different particle sizes $a$ and wavelengths $\lambda$, the wavelengthdependent opacity $\kappa_{\lambda, a}$ for each particle size per gram of dust is given by

$$
\kappa_{\lambda, a}=\frac{\pi a^{2} Q_{\mathrm{ext}}(\lambda, a)}{4 \pi \rho_{s} a^{3} / 3}
$$

To calculate the opacity per gram of dust in the protoplanetary disk, we need the normalized size distribution of the grains at a specific location. We utilize the mass density distribution of dust, $\Sigma_{\mathrm{d}}(r, a)$, calculated in logarithmic bins of grain size using twopoppy. The opacity per gram of dust in the protoplanetary disk is then obtained using

$$
\kappa_{\lambda}=\frac{\int \kappa_{\lambda, a} \Sigma_{\mathrm{d}}(r, a) \mathrm{d} \ln a}{\int \Sigma_{\mathrm{d}}(r, a) \mathrm{d} \ln a} .
$$

This wavelength-dependent opacity is used to calculate the Rosseland mean opacity per gram of dust,

$$
\frac{1}{\kappa_{R}}=\frac{\int_{0}^{\infty}\left(1 / \kappa_{\lambda}\right)\left(\partial B_{\lambda} / \partial T\right) d \lambda}{\int_{0}^{\infty}\left(\partial B_{\lambda} / \partial T\right) d \lambda},
$$

where $B_{\lambda}$ is the Planck function and $T$ is the temperature used in our protoplanetary disk model. To obtain the Rosseland

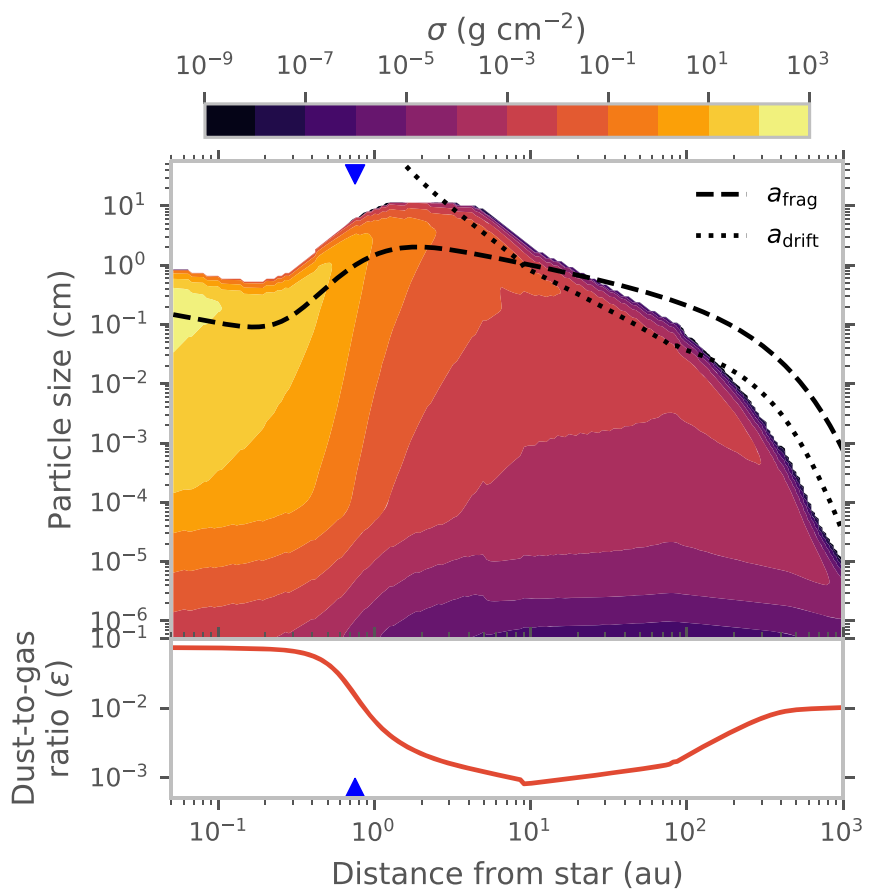

Figure 1. Size distribution and dust-to-gas ratio $(\epsilon)$ at time $t=1 \mathrm{Myr}$ for a twopoppy simulation with variable $v_{\text {frag }}$ and $\alpha_{\mathrm{t}}=10^{-3}$. The size limits imposed by fragmentation and drift are shown with dashed and dotted lines in the upper panel. The location of the ice line is marked with blue triangles.

mean opacity per gram of protoplanetary disk material, we multiply the $\kappa_{R}$ obtained above by the local dust-to-gas ratio, $\epsilon=\Sigma_{d}(r) / \Sigma_{g}(r)$, of the disk. We do not include the gas opacity in our calculations, as the dust opacity dominates even in the regions with the lowest dust-to-gas ratio and/or largest particle sizes (see Section 4.1).

\section{Dust Opacity in Protoplanetary Disks}

\subsection{Opacity from a Simulated Size Distribution}

In this section, we focus on quantifying the changes in the dust opacity due to location-dependent variations in the dust size distribution. We show the full radially varying twopoppy size distribution in the top panel of Figure 1 and the resulting Rosseland mean opacity per gram of dust in Figure 2 . The size distribution in the inner 10 au is dominated by coagulationfragmentation equilibrium, while the increase in $v_{\text {frag }}$ beyond the water-ice line at $\sim 1$ au manifests as an increase in the maximum grain size $\left(a_{\text {frag }} \propto v_{\text {frag }}^{2}\right.$ from Equation (8a)). Since larger grains contain more mass and the size distribution is slightly top-heavy, this increase in $v_{\text {frag }}$ causes the surface density of small grains $(\lesssim 10 \mu \mathrm{m})$ to decrease by multiple orders of magnitude. Because these grains contribute significantly to $\kappa_{\mathrm{R}}$, this change is responsible for the factor of $\sim 5$ decrease in the simulated $\kappa_{\mathrm{R}}$ shown in Figure 2. Beyond $\sim 10 \mathrm{au}$, the maximum grain size is set by radial drift of the large grains instead of fragmentation as particles drift inward before they can grow to the fragmentation barrier. Without fragmentation to replenish the supply of small grains, the size distribution in this region becomes more top-heavy relative to the distribution produced by the coagulation-fragmentation equilibrium in the inner disk. The $\kappa_{\mathrm{R}}$ in this cold outer disk region is dominated by larger grains $(\sim 100 \mu \mathrm{m})$ that are 


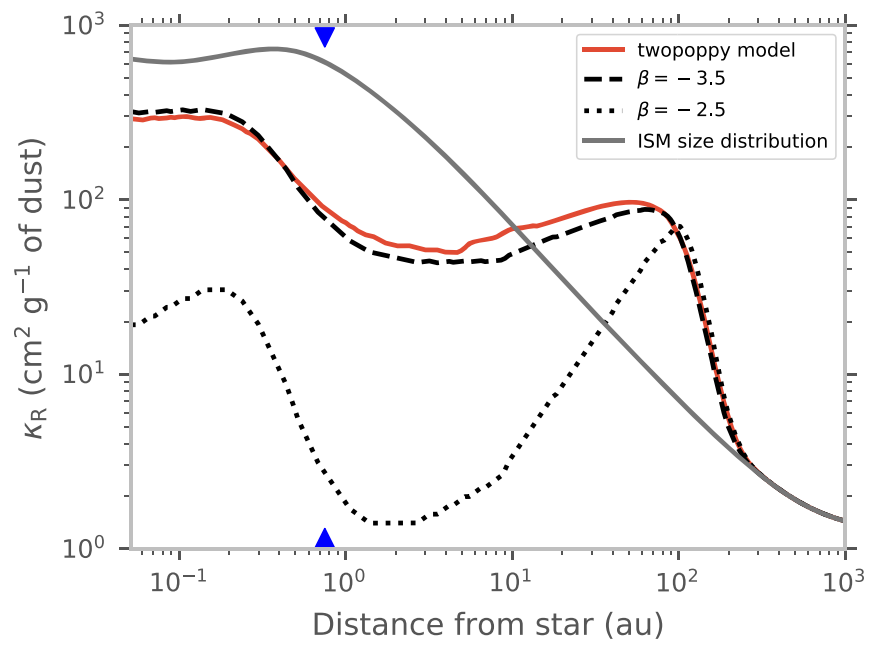

Figure 2. Rosseland mean opacity per gram of dust as a function of distance from the star at time $t=1 \mathrm{Myr}$. We adopt $\alpha_{\mathrm{t}}=10^{-3}$ and a variable $v_{\text {frag }}$ that changes across the water-ice line for our twopoppy model. For the power-law distributions, $a_{\max }$ is set by the location-specific maximum grain size calculated from twopoppy, which is given by Equation (8a) (fragmentation-limited), Equation (10) (drift-limited), or the growth-timescale limit. The location of the ice line is marked with blue triangles.

relatively abundant, leading to a modest increase in the simulated $\kappa_{\mathrm{R}}$, as shown in Figure 2.

In Figure 2, we compare $\kappa_{\mathrm{R}}$ for a size distribution simulated by twopoppy at time $t=1 \mathrm{Myr}$ with three different grain size distributions: the ISM size distribution $\left(\beta=-3.5, a_{\max }=0.25\right.$ $\mu \mathrm{m})$ and power-law distributions with $\beta$ of either -2.5 or -3.5 and maximum particle sizes set to the fragmentation (Equation (8a)), radial drift (Equation (10)), or growth-timescale limits, as appropriate. We find that the dust opacity for the simulated size distribution differs significantly from that of the ISM size distribution (see also Savvidou et al. 2020). The opacity of the ISM size distribution only varies as a consequence of the decreasing temperature in the disk. In contrast, opacity from the simulated size distribution reflects radially varying grain growth and transport processes in the disk (e.g., Akimkin et al. 2020 made the same observation). It is noteworthy that a power-law distribution with $\beta=-3.5$ (the same as that of the ISM) and $a_{\max }$ set by the relevant physics of fragmentation and radial drift yields a $\kappa_{\mathrm{R}}$ profile that is in good agreement with the simulated results.

We illustrate the effect of the maximum grain size $a_{\max }$ and power-law index $\beta$ on $\kappa_{\mathrm{R}}$ in Figure 3. The smallest value of $a_{\max }$ shown on the plot corresponds to the ISM size distribution. For top-heavy distributions with $\beta>-4$, most of the mass is concentrated in the larger dust grains. Increasing $a_{\max }$ therefore redistributes the dust mass from smaller grains to larger grains, reducing the total number of small grains. This can significantly alter the overall opacity of the dust; if we compare $\kappa_{\mathrm{R}}$ for $a_{\max }=0.1 \mathrm{~cm}$ (which is more typical for dust in a disk) and $\beta=-3.5$ with the equivalent ISM value, it is almost 20 times larger at $10 \mathrm{~K}$. Conversely, this same depletion of smaller grains for $a_{\max }=0.1 \mathrm{~cm}$ means that $\kappa_{\mathrm{R}}$ is half the corresponding ISM value at $1000 \mathrm{~K}$. Using a realistic $a_{\max }$ for the power-law size distribution of dust in a protoplanetary disk therefore leads to a reduced $\kappa_{\mathrm{R}}$ in the hotter inner disk and an enhanced $\kappa_{\mathrm{R}}$ in the colder outer disk.

In contrast to this result, the opacity from a power-law size distribution with $\beta=-3.5$ and $a_{\max }$ set by Equations (8a) and

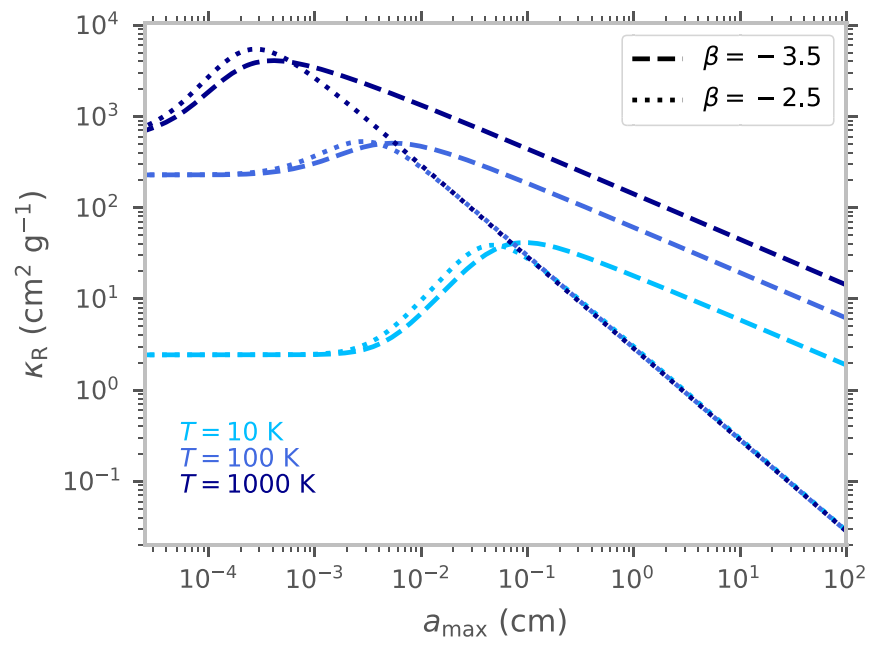

Figure 3. Rosseland mean opacity per gram of dust for a power-law grain size distribution with $\beta=-3.5$ and -2.5 and three different temperatures. The lowest value of $a_{\max }=0.25 \mu \mathrm{m}$ on this plot is the commonly adopted value for the ISM size distribution.

(10) and growth timescale $\tau_{\text {grow }}$ provides a relatively good match to the opacity from the full simulated size distribution. The power-law size distribution with $\beta=-2.5$ does not perform as well; this is due to the top-heaviness of the $\beta=-2.5$ size distribution, which leads to a dramatic depletion in the number of small grains. Since the small grains that contribute most significantly to $\kappa_{\mathrm{R}}$ at the protoplanetary disk temperatures are absent, the opacity for $\beta=-2.5$ is $\gtrsim 1$ order of magnitude lower than that for our twopoppy simulation. These results for different $\beta$ values are similar to previous findings for the dust opacity at specific wavelengths (e.g., D'Alessio et al. 2001).

\subsection{Opacity from a Radially Varying Dust-to-gas Ratio}

Now that we have explored the effect of a radially varying dust size distribution on the Rosseland mean opacity per gram of dust, we can account for the radially varying dust-to-gas ratio $\epsilon$. As noted earlier, we assume that the contribution of the gas opacity to $\kappa_{\mathrm{R}}$ is negligible. The dust-to-gas ratio (or metallicity) is typically fixed to a single global value (e.g., Bitsch et al. 2015; Mordasini 2018). However, this ratio can change radially as dust abundance evolves. Here we use our simulations to explore how the distribution of dust evolves in time as a function of assumed disk properties such as the turbulence strength $\alpha_{\mathrm{t}}$ and $v_{\text {frag. }}$.

We begin our simulation with a globally uniform $\epsilon=0.01$ and show the resulting vertically integrated dust-to-gas ratio $\left(\epsilon=\Sigma_{\mathrm{d}} / \Sigma_{\mathrm{g}}\right)$ at time $t=1 \mathrm{Myr}$ for our fiducial model in the bottom panel of Figure 1. As grains begin to grow and their Stokes number increases, they face a stronger headwind from the gas and start drifting toward the star (see Birnstiel et al. 2012 for a more detailed discussion on how the dust-to-gas ratio evolves in the disk). In the outermost regions of the disk $(\gtrsim 100 \mathrm{au}$ ), the grain growth rate is so slow that particles do not reach the drift barrier, i.e., they do not drift very efficiently. The $\epsilon$ far out does not evolve significantly and only decreases slowly as one moves closer to $100 \mathrm{au}$. Between $\sim 10$ and $100 \mathrm{au}$, grains drift inward faster than they can grow, causing the dust-to-gas ratio to decrease over time. In the inner disk, orbital timescales are shorter and grain growth is rapid. This 


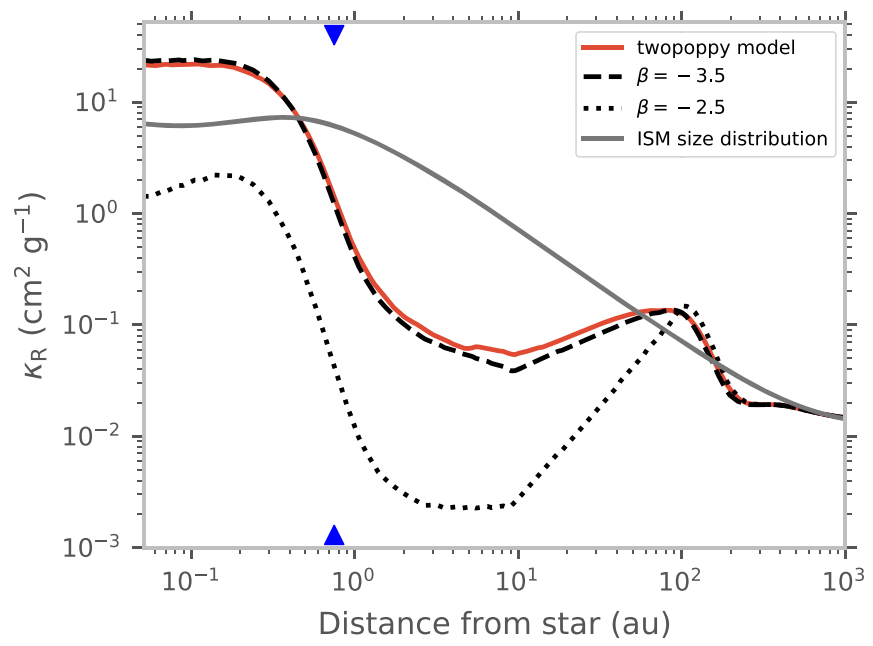

Figure 4. Rosseland mean opacity per gram of protoplanetary disk material at time $t=1 \mathrm{Myr}$ and for a variable $v_{\text {frag }}$ and $\alpha_{\mathrm{t}}=10^{-3}$. This plot is similar to Figure 2 except that the opacity per gram of dust is multiplied by the radially varying dust-to-gas ratio. For the ISM size distribution, the dust-to-gas ratio is assumed to be 0.01 everywhere. The location of the ice line is marked with blue triangles.

means that grains reach the fragmentation barrier before they can drift appreciably. For a fixed $v_{\text {frag }}$ and $\alpha_{\mathrm{t}}$, the Stokes number of the largest grains also decreases as one moves closer to the star (see Equation (8a)). This means that grains in the fragmentation-dominated inner disk are better coupled to the gas, and the dust-to-gas ratio does not decline as rapidly as in the drift-dominated outer disk region. In fact, the dust-to-gas ratio in the inner disk may even be enhanced by the migration of dust from the outer disk.

Depending on the magnitude of the velocity offset, the change in $v_{\text {frag }}$ across the ice line can have a dramatic effect on the dust dynamics. When large grains drifting inward from the outer disk cross the ice line, they lose their ice, and their fragmentation velocity decreases to the value characteristic of ice-free dust. Post fragmentation grains are therefore smaller, and their St is reduced, slowing their inward drift and causing a pileup of dust inside the ice line. The magnitude of this effect can be quite large; for a factor of 10 decrease in $v_{\text {frag }}$ across the ice line, the St of the largest grains decreases by almost 2 orders of magnitude $\left(\mathrm{St}_{\text {frag }} \propto v_{\text {frag }}^{2}\right)$. As shown in Figure 1, this enhances the dust-to-gas ratio $\epsilon$ within $\sim 1$ au by almost an order of magnitude at $t=1 \mathrm{Myr}$ relative to the starting $\epsilon$ of 0.01 . Conversely, most of the disk beyond 1 au is significantly depleted of dust with $\epsilon \sim 10^{-3}$ for a large part of the outer disk. The effect of radial drift, fragmentation, and a change in $v_{\text {frag }}$ across the ice line on dust dynamics has been extensively described in Birnstiel et al. (2010) and Pinilla et al. (2017), and we refer the reader to these studies for a comprehensive exploration of this topic.

We can use this radially and temporally varying dust-to-gas ratio to update our calculation of the Rosseland mean opacity of the disk. Figure 4 shows the $\kappa_{\mathrm{R}}$ per gram of protoplanetary disk material for our simulated size distribution. This plot is the same as Figure 2 except that the $\kappa_{\mathrm{R}}$ profiles shown in that figure are now multiplied by the dust-to-gas ratio. We plot the ISM $\kappa_{\mathrm{R}}$ assuming a constant dust-to-gas ratio of 0.01 in order to better illustrate the differences between our model and the widely used ISM opacity model. Within the ice line, the dustto-gas ratio is enhanced by a factor of 10 relative to the ISM model, which partially compensates for the reduction in opacity due to the increased grain sizes (Figure 2). As we move beyond the ice line, the decreasing quantity of dust and increasing concentration of dust mass in larger particle sizes lead to a steep decline in the opacity. Our $\kappa_{\mathrm{R}}$ between $\sim 1$ and $\sim 10$ au is smaller than the ISM value by more than a factor of 10 .

\subsection{Dependence on the Assumed Fragmentation Velocity and Turbulence Strength}

Our fiducial model predicts that the dust opacity will decrease by more than 2 orders of magnitude as we move outside the ice line. However, the magnitude of this gradient depends strongly on the absolute and relative efficiency of dust transport in the inner and outer disk. The transition from the fragmentation-dominated to the drift-dominated regime can be expressed as a function of the fragmentation velocity $v_{\text {frag }}$ and the turbulence strength $\alpha_{\mathrm{t}}$ (e.g., Birnstiel et al. 2015):

$$
\frac{v_{\text {frag }}^{2}}{v_{\mathrm{K}}^{2}}>\frac{3 \alpha_{\mathrm{t}} \epsilon}{\gamma} \text {. }
$$

This transition also depends on the Keplerian velocity $v_{\mathrm{K}}$, the dust-to-gas ratio $\epsilon$, and $\gamma=|\mathrm{d} \ln P / \mathrm{d} \ln r|$. All of these quantities can vary as a function of $r$ (although we assume $\alpha_{\mathrm{t}}$ is constant in our work), and in regions where this inequality is satisfied, the disk becomes drift-dominated. Since $\alpha_{\mathrm{t}}$ and $v_{\text {frag }}$ are not known a priori, we run a grid of models over $\alpha_{\mathrm{t}} \in\left[10^{-4}, 10^{-3}, 10^{-2}\right]$, where $\alpha_{\mathrm{t}}=10^{-3}$ is our fiducial, and $v_{\text {frag }}=0.1-10 \mathrm{~m} \mathrm{~s}^{-1}$ for ice-free grains and $1-50 \mathrm{~m} \mathrm{~s}^{-1}$ for icy grains (e.g., Blum \& Wurm 2008; Gundlach \& Blum 2015). We consider all possible combinations of these two fragmentation velocities as long as they meet the requirement that $v_{\text {frag }}$ for icy grains is greater than or equal to $v_{\text {frag }}$ for ice-free grains. ${ }^{6}$

Figure 5 shows the Stokes number of the largest grains $\mathrm{St}_{\max }$, the dust-to-gas ratio $\epsilon$, and the disk's Rosseland mean opacity $\kappa_{\mathrm{R}}$ for this grid of models. As $\alpha_{\mathrm{t}}$ decreases, we find that $\epsilon$ varies more strongly with orbital distance, a consequence of the difference in the absolute values of $\mathrm{St}_{\max }$ for different $\alpha_{\mathrm{t}}$. For $\alpha_{\mathrm{t}}=10^{-4}, \mathrm{St}_{\max } \gtrsim 10^{-2}$ between $\sim 1$ and $100 \mathrm{au}$. A larger Stokes number beyond the ice line leads to a more efficient inward drift of dust from the outer to the inner disk. For lower $\alpha_{\mathrm{t}}$, the transition to the drift-dominated region also happens closer to the star (see Equation (16) above), creating a "kink" in the $\mathrm{St}_{\max }$ and $\epsilon$ profiles (e.g., at $10 \mathrm{au}$ in our fiducial model; see bottom panel of Figure 1). In the outer disk, all models that transition to the drift-dominated regime converge to similar values for $\mathrm{St}_{\max }$ and $\epsilon$. For $\alpha_{\mathrm{t}}=10^{-2}$, this transition moves outside $\sim 100$ au for most models, causing the disk to be globally fragmentation-dominated. As a result, $\mathrm{St}_{\max }$ has a lower value throughout the disk, and dust migration is suppressed.

The high $\mathrm{St}$ in the low $\alpha_{\mathrm{t}}$ disk model, which aids the radial transport of dust grains in the outer disk, can also potentially diminish the dust pileup in the inner disk. Since $\mathrm{St}_{\text {frag }} \propto \alpha_{\mathrm{t}}^{-1}$, $\mathrm{St}_{\max }$ in the inner disk is larger for lower $\alpha_{\mathrm{t}}$. As long as $\mathrm{St}_{\max }<1$, the inward drift velocity will be larger for a larger value of $\mathrm{St}_{\text {max }}$. This means that dust grains in the inner disk will

\footnotetext{
6 We note that the twopoppy models are calibrated with the full numerical models for a smaller range of $v_{\text {frag }}\left(1-10 \mathrm{~m} \mathrm{~s}^{-1}\right)$ than we study here. However, this should not be a major concern, as the underlying collisional outcome model is the same.
} 


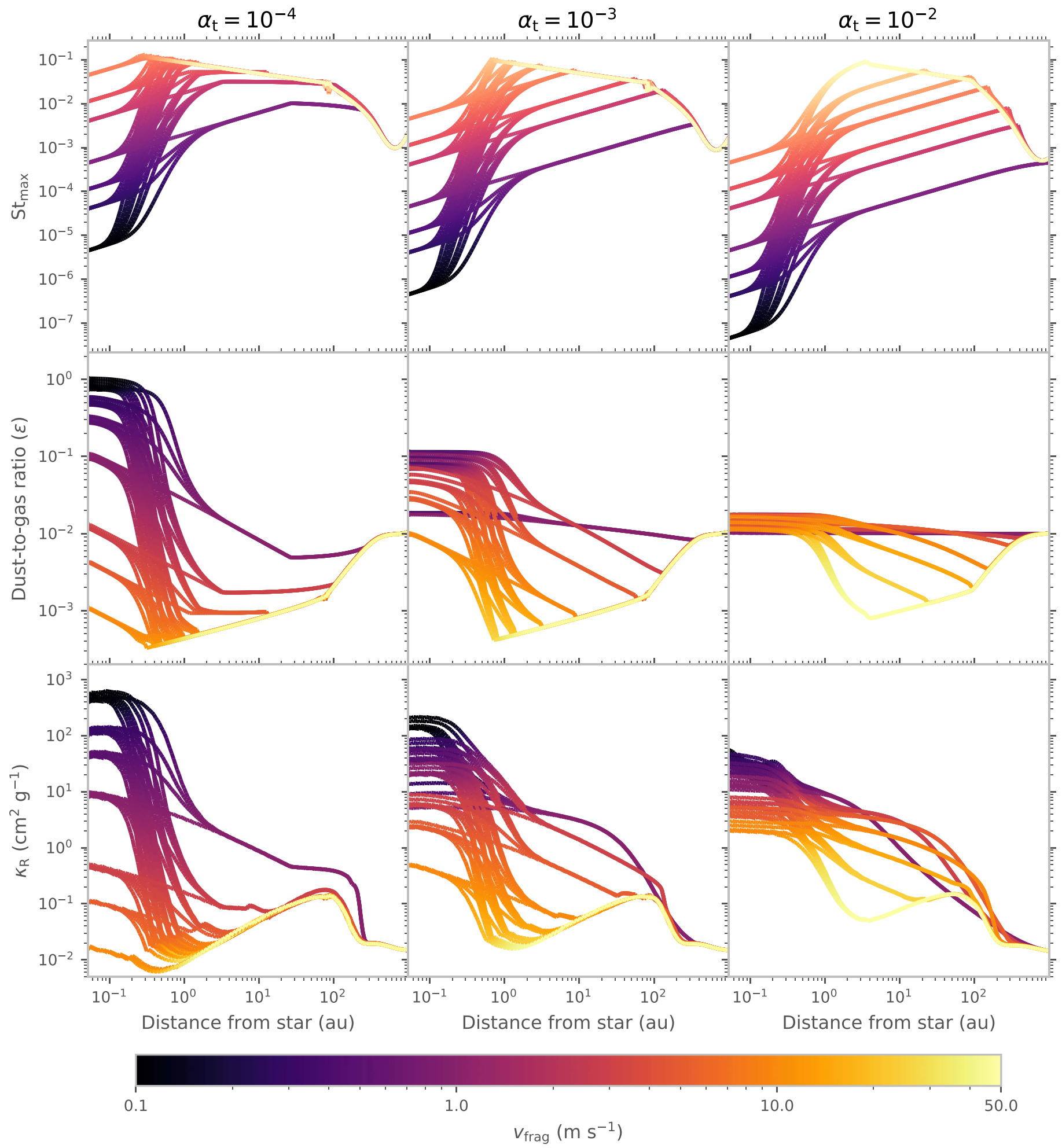

Figure 5. Stokes number of the largest grain size $\left(\mathrm{St}_{\mathrm{max}}\right)$, dust-to-gas ratio $(\epsilon)$, and Rosseland mean opacity per gram of protoplanetary disk material for a range of fragmentation velocities within and beyond the water-ice line, as well as three different turbulence strengths after 1 Myr of evolution. The fragmentation velocity $v_{\text {frag }}$ takes values in the range $0.1-10 \mathrm{~m} \mathrm{~s}^{-1}$ for ice-free grains and $1-50 \mathrm{~m} \mathrm{~s}^{-1}$ for icy grains (Blum \& Wurm 2008; Gundlach \& Blum 2015). The ice line is located at 0.75 au in all of our models.

move inward faster when $\alpha_{\mathrm{t}}$ is lower, reducing the timescale over which dust is depleted in the inner disk and preventing a pileup of dust drifting in from the outer disk. This is evident in the middle panel of Figure 5, which shows that when $\alpha_{\mathrm{t}}=10^{-4}$, the dust-to-gas ratio in the inner disk can be either very high $\left(\sim 1\right.$, efficient pileup, low $\left.v_{\text {frag }}\right)$ or very low $\left(\sim 10^{-3}\right.$, no pileup, high $v_{\text {frag }}$ ), depending on the assumed fragmentation velocities. Maximizing the dust-to-gas ratio and, consequently, the opacity gradient in the radial direction therefore requires an intermediate value of $\alpha_{\mathrm{t}}$, which in turn is dependent on $v_{\mathrm{frag}}$.

Larger differences in the $v_{\text {frag }}$ values for icy and ice-free grains lead to a larger change in $\mathrm{St}_{\max }$ across the ice line. This 


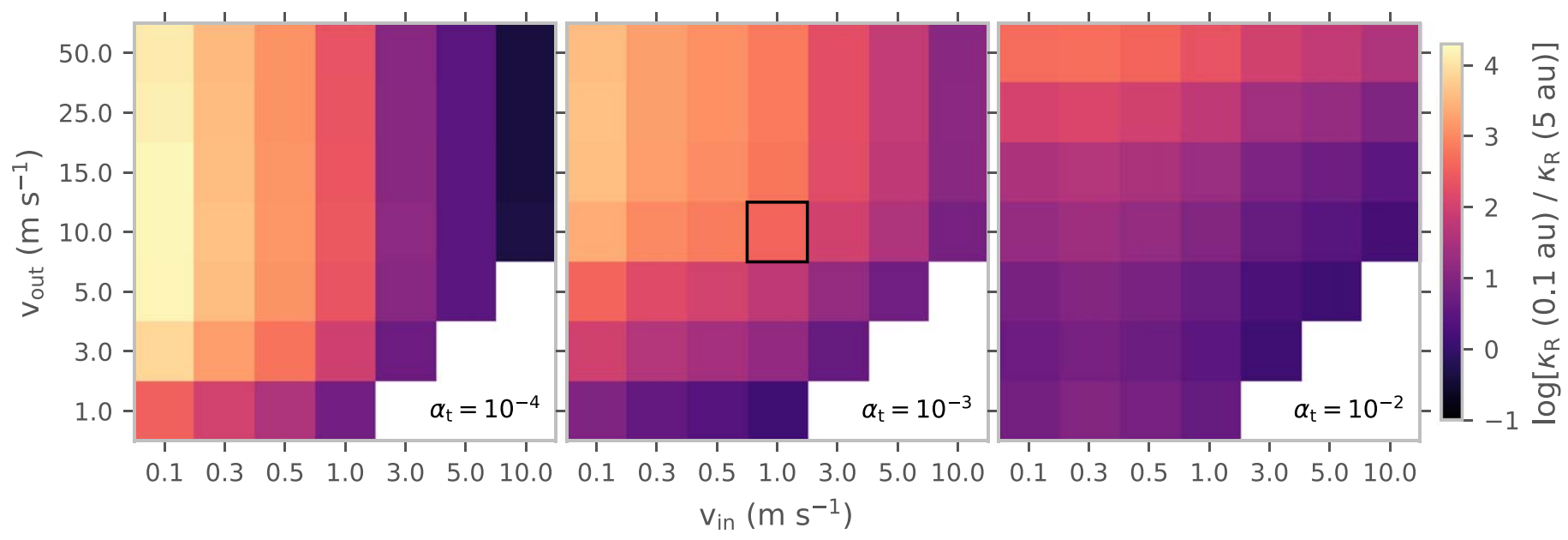

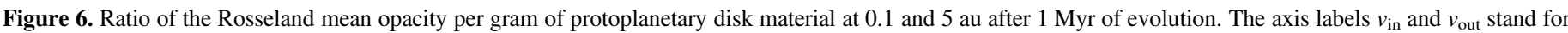
the fragmentation velocity within and beyond the ice line. Our fiducial model is outlined with a black square.

in turns results in a depletion of dust in the outer disk and a pileup of dust in the inner disk, leading to a larger opacity contrast between the inner and the outer disk (see Figure 6), as long as the value of $\alpha_{\mathrm{t}}$ does not nullify these effects by producing either globally low values of $\mathrm{St}_{\max }$ (well-coupled dust and little dust transport) or large values of $\mathrm{St}_{\max }$ within the ice line (dust drifts toward the star and does not pile up). However, when $v_{\text {frag }}$ is large everywhere in the disk (e.g., $10 \mathrm{~m} \mathrm{~s}^{-1}$ for ice-free grains and $50 \mathrm{~m} \mathrm{~s}^{-1}$ for icy grains), particles will have large $\mathrm{St}_{\max }$ and rapidly drain onto the star.

To simplify comparisons between models, in Figure 6, we focus on the ratio of the disk opacity $\kappa_{\mathrm{R}}$ at 0.1 and $5 \mathrm{au}$. These distances are chosen to best capture the opacity contrast for the full set of disk models; they are also approximately where subNeptunes and gas giants are most numerous, respectively. We find that there is a large range of choices for $v_{\text {frag }}$ and $\alpha_{\mathrm{t}}$ that lead to opacity contrasts that are equal to or larger than the one in our fiducial model. Decreasing $\alpha_{\mathrm{t}}$ enlarges $\mathrm{St}_{\max }$ and accelerates the grain radial transport, enhancing the contrast in the opacity across the ice line. Larger differences in $v_{\text {frag }}$ between icy and ice-free grains also produce greater opacity contrasts, as they lead to a strong gradient in dust transport efficiency across the ice line.

The opacity contrast with increasing $v_{\text {frag }}$ for icy grains saturates at a value that depends on the $v_{\text {frag }}$ for ice-free grains. This is most evident in the lower $\alpha_{\mathrm{t}}$ models and occurs because $\mathrm{St}_{\max }$ and $\epsilon$ converge to similar values in the outer disk (Figure 5). Beyond this limit, increasing the $v_{\text {frag }}$ for icy grains does not lead to an increase in the Stokes number of the largest grains in the outer disk but instead simply pushes the transition from the fragmentation-dominated regime to the drift-dominated regime inward. This limits the supply of dust from the outer disk and causes the opacity contrast to saturate at a fixed $v_{\text {frag }}$ for ice-free grains.

Recent observations of protoplanetary disks appear to favor values for $\alpha_{\mathrm{t}}$ that are lower than $10^{-2}$ (e.g., Pinte et al. 2016; Flaherty et al. 2018). As we discussed earlier, it is less clear how large the difference in $v_{\text {frag }}$ for icy and ice-free dust grains may be (Gundlach et al. 2018; Steinpilz et al. 2019; Kimura et al. 2020). However, our parameter space exploration suggests that there is a wide range of plausible scenarios that can lead to a large opacity gradient between the inner and outer disk regions.

\subsection{Dust Opacity in a 3D Disk}

So far, we have only considered vertically integrated disk models. In this section, we examine the vertical structure of the dust distribution and its potential importance for planet formation (e.g., polar accretion of gas onto planetary cores; Fung et al. 2015; Ormel et al. 2015; Cimerman et al. 2017; Lambrechts \& Lega 2017) and modeling protoplanetary disks. The vertical structure of gas and dust is controlled by a complicated coupling between the disk temperature, opacity, and turbulence. Self-consistently taking these couplings into account is beyond the scope of our study; instead, we utilize a simple vertically isothermal disk model. Even with this simplification, our model produces a nonuniform vertical distribution of dust grains.

We use the prescribed radial temperature structure from Equation (3) and assume a vertically isothermal disk structure in order to calculate the vertical structure of the dust and gas. Under this assumption, the gas density $\rho_{g} \propto e^{-z^{2} / H_{g a s}^{2}}$, where $z$ is the height from the midplane and $H_{\mathrm{gas}}=c_{s} / \Omega$ is the gas disk scale height. For the vertical dust density distribution, we utilize the expression obtained by Fromang \& Nelson (2009) for the steady-state distribution of dust (Equation (11)). We calculate the 3D dust density $\rho_{d}(z, a)$ for logarithmically binned grain sizes and sum it to obtain the total dust density $\rho_{d}(z)$. The dust-to-gas ratio $\epsilon$ is then simply calculated as $\rho_{d} / \rho_{g}$.

The top panel of Figure 7 shows the resulting dust-to-gas ratio $\epsilon$ as a function of $z$ and distance from the star for our fiducial model at a disk age of $1 \mathrm{Myr}$. The differences in $\epsilon$ as a function of $z$ within and beyond the ice line can be understood by examining the Stokes number of the largest grains $\mathrm{St}_{\max }$ present in each region of the disk (bottom panel of Figure 7). Within $\sim 1 \mathrm{au}, \mathrm{St}_{\max }$ can fall down to $\sim 10^{-4}$. These particles will be vertically well mixed with the gas-i.e., the scale height of the dust grains is comparable to that of the gas-flattening the vertical gradient in the dust-to-gas ratio. However, outside the ice line, large grains with $\mathrm{St}_{\max } \gtrsim 10^{-2}$ are present. These grains are concentrated near the midplane and constitute most of the dust mass budget, resulting in a steep vertical gradient in $\epsilon$. Figure 8 shows the Rosseland mean opacity of the disk as a function of height from the midplane and distance from the star. As expected, we find that the disk opacity is essentially independent of $z$ within the ice line. In contrast, the concentration of large grains near the midplane beyond the ice line leads to a decline in disk opacity as a function of $z$. 


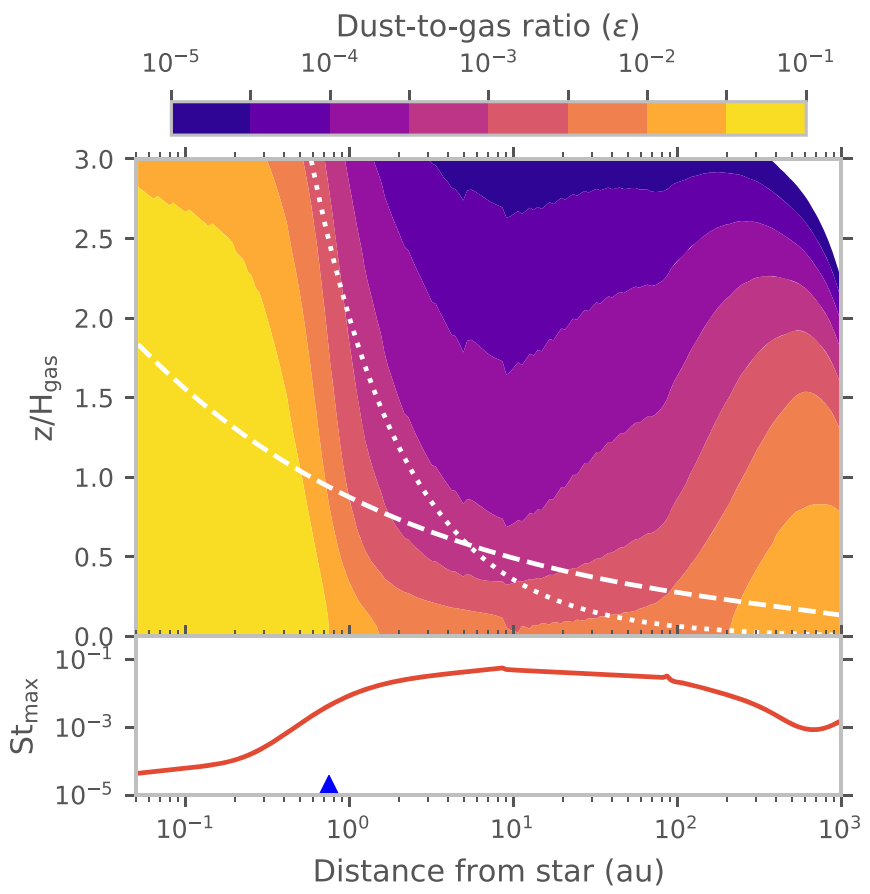

Figure 7. The top panel shows the dust-to-gas ratio $\epsilon$ as a function of height above the midplane $z$ and distance from the star after $1 \mathrm{Myr}$ of evolution. The white dashed and dotted lines mark the height of the Hill radius $R_{\text {Hill }}$ and Bondi radius $R_{\text {Bondi }}$ of a $15 M_{\oplus}$ planet, respectively. The bottom panel shows the midplane Stokes number of the largest grains present in the disk at $t=1 \mathrm{Myr}$. The water-ice line is marked with a blue triangle. Well-coupled grains within the ice line lead to efficient vertical mixing of grains and hence a weak dependence of $\epsilon$ on $z$. Beyond the ice line, large grains that dominate the dust mass settle close to the midplane, which leads to a strong decline in $\epsilon$ as a function of $z$.

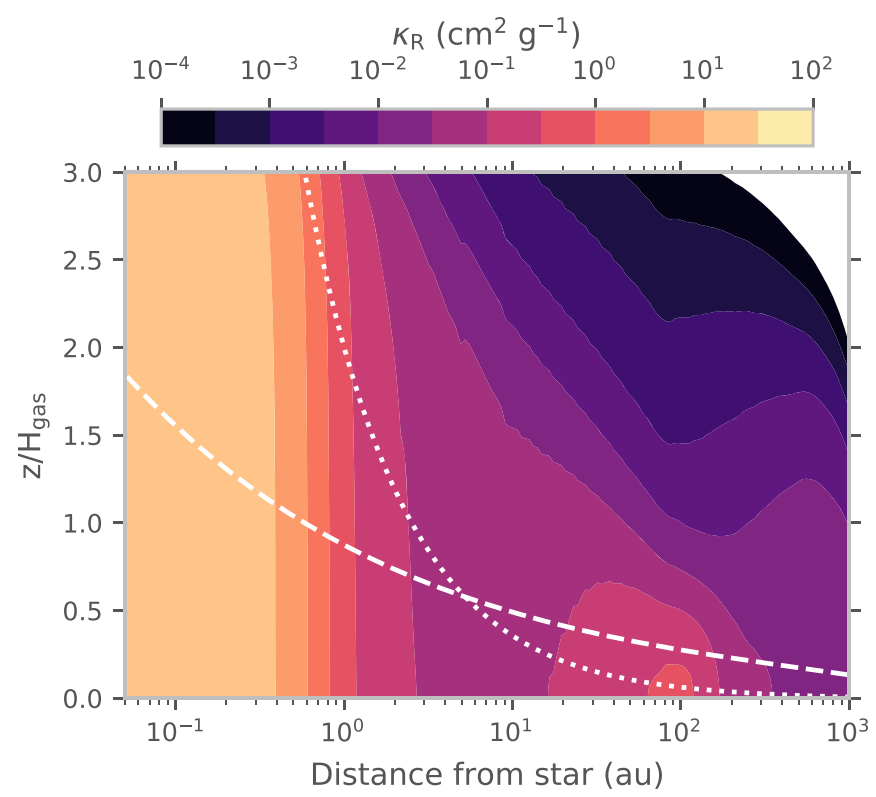

Figure 8. Rosseland mean opacity per gram of protoplanetary disk material as a function of height above the midplane $z$ and distance from the star after $1 \mathrm{Myr}$ of evolution. The white dashed and dotted lines mark the height of the Hill radius $R_{\text {Hill }}$ and Bondi radius $R_{\text {Bondi }}$ of a $15 M_{\oplus}$ planet, respectively. Vertically well-mixed dust within the ice line leads to little variation in $\kappa_{\mathrm{R}}$ as a function of $z$. Grain settling and a strong decline in $\epsilon$ with $z$ lead to a gradient in $\kappa_{\mathrm{R}}$ as a function of $z$ beyond the ice line.

We mark the Hill radius $R_{\text {Hill }}=a\left(M_{\mathrm{p}} / 3 M_{*}\right)^{1 / 3}$ and Bondi radius $R_{\text {Bondi }}=G M_{\mathrm{p}} / c_{s}^{2}$ of a $15 M_{\oplus}$ core with dashed and dotted lines, respectively, in Figures 7 and 8. We choose a mass

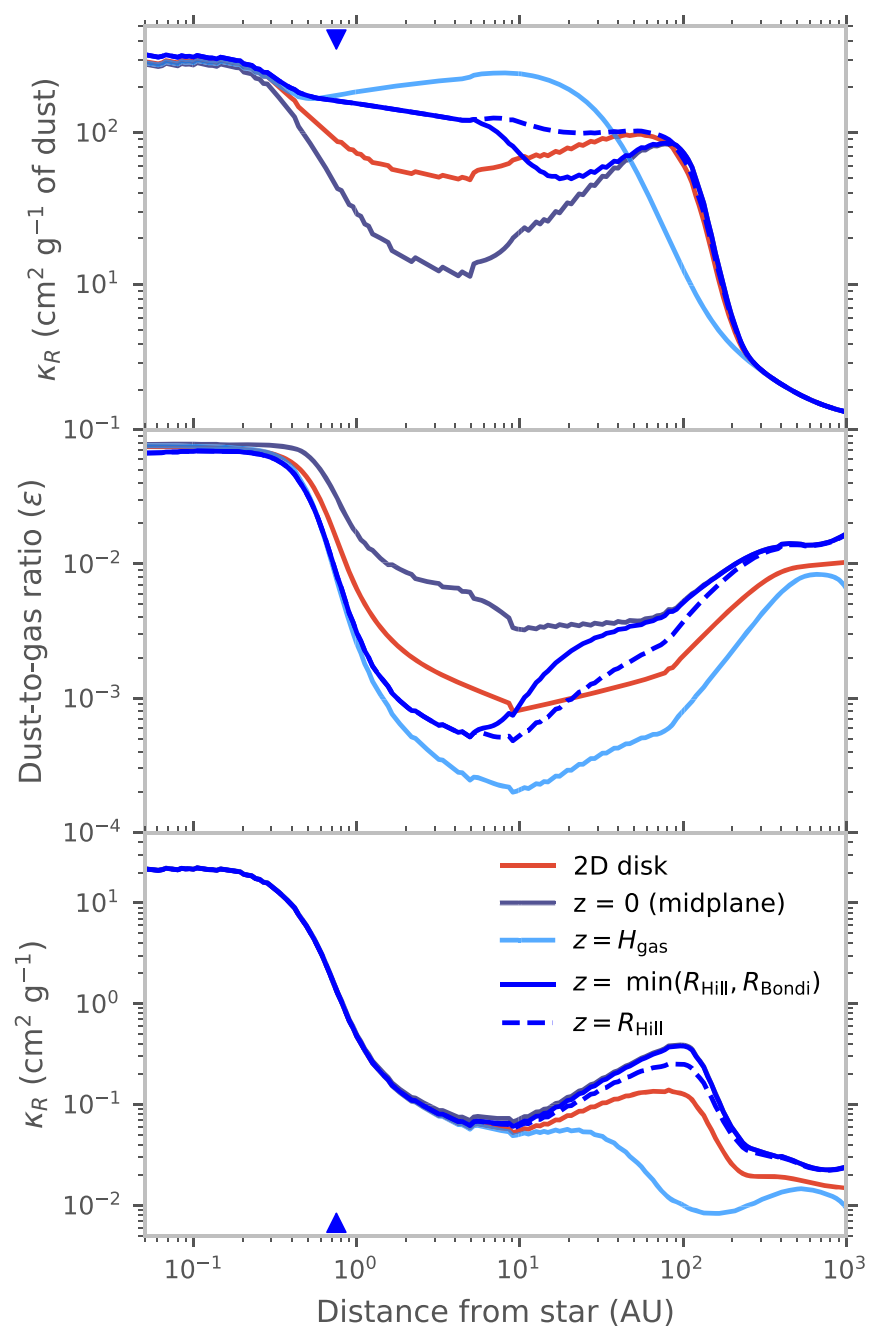

Figure 9. Comparison of the Rosseland mean opacity per gram of dust, dust-togas ratio $\epsilon$, and Rosseland mean opacity per gram of protoplanetary disk material $\kappa_{\mathrm{R}}$ for our fiducial 2D disk-integrated model and 3D disk model after $1 \mathrm{Myr}$ of evolution. We plot the values of these quantities in the disk midplane $(z=0)$, a single gas scale height above the midplane $\left(z=H_{\text {gas }}\right)$, and at heights of a $15 M_{\oplus}$ planet's $R_{\mathrm{Hill}}$ and $\min \left(R_{\mathrm{Hill}}, R_{\mathrm{Bondi}}\right)$ above the midplane. The waterice line is marked with blue triangles.

of $15 M_{\oplus}$ as our fiducial case, as it is representative of a giant planet core. Planetary cores close to thermal or superthermal mass (equivalently, $R_{\text {Hill }} \leqslant R_{\text {Bondi }}$ ) are expected to accrete gas from heights on the order of the Hill radius (e.g., Lambrechts \& Lega 2017). For subthermal cores (equivalently, $R_{\text {Hill }}>R_{\text {Bondi }}$, on the other hand, the natural length scale is expected to be the Bondi radius (see, e.g., the subthermal cases of Ormel et al. 2015 and Fung et al. 2019). The exact origin height of the accretion flow is unclear given how unsteady the flow morphology is in $3 \mathrm{D}$ calculations. In this work, we assume that the material accreted by the planet is well represented by the properties of dust and gas present at $\min \left(R_{\text {Hill }}, R_{\text {Bondi }}\right)$ above the disk midplane. In Section 4.1, we show the effect of varying this height on the calculated gas-to-core mass fraction of a planet.

Figure 9 highlights how the radial profile of the dust-to-gas ratio and dust opacity differ for different heights above the disk midplane: $z=0$ (disk midplane), $z=H_{\mathrm{gas}}$, and $z=R_{\mathrm{Hill}}$ and $z=\min \left(R_{\text {Hill }}, R_{\text {Bondi }}\right)$ for a $15 M_{\oplus}$ core. We also provide a calculation of the vertically integrated disk model for comparison. In the top panel, we plot $\kappa_{\mathrm{R}}$ per gram of dust, which 

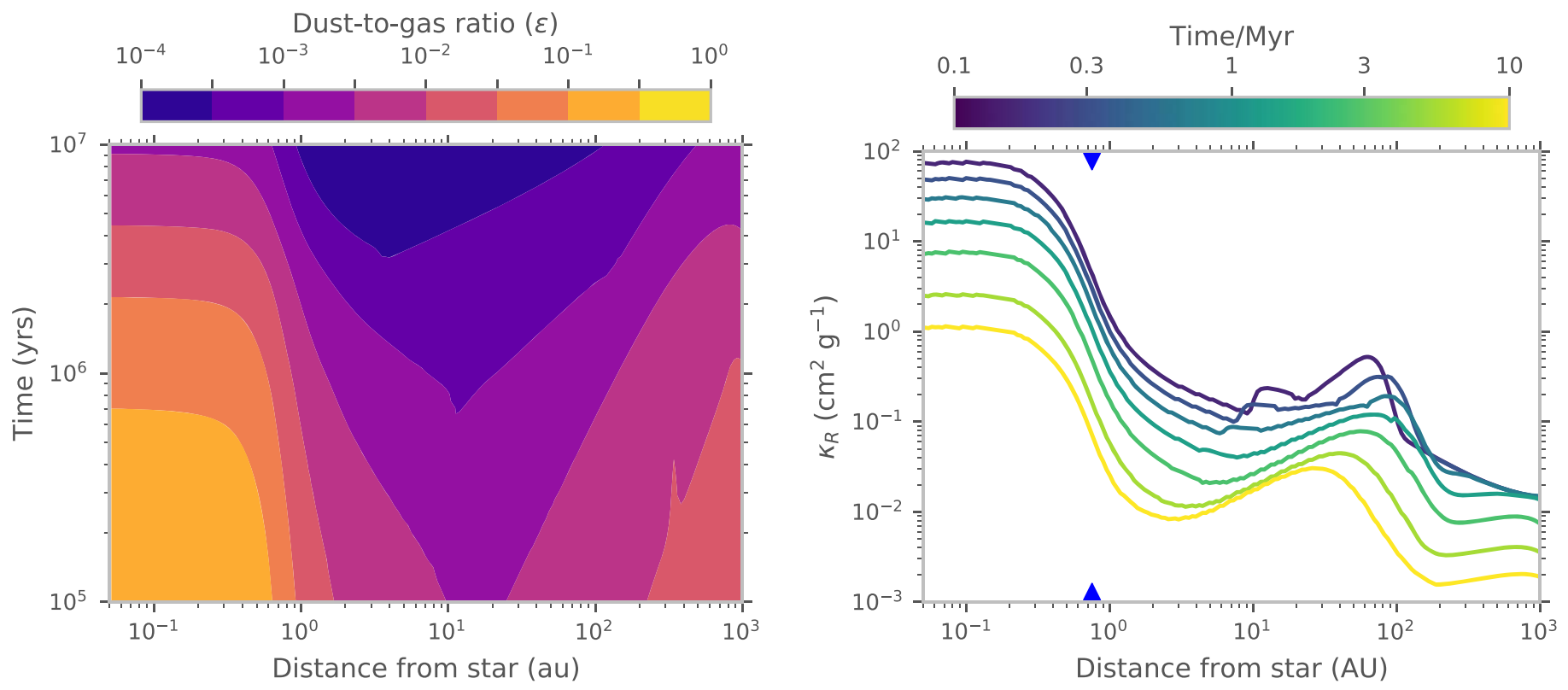

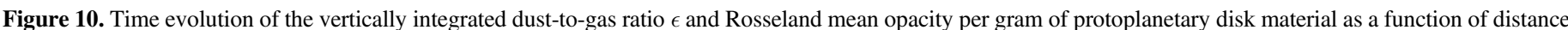

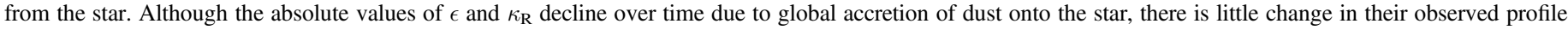

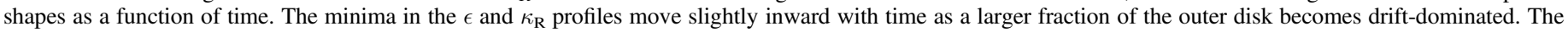
water-ice line is marked with blue triangles in the right panel.

depends only on the local size distribution of the dust. The features present in the $\kappa_{\mathrm{R}}$ profiles result from changes in the relative abundances of the grain sizes that contribute most to the opacity at the local temperature. In the disk midplane beyond the ice line, most of the opacity contribution comes from grains that are $10-100 \mu \mathrm{m}$ in size, but most of the mass (per gram of dust) resides in grains that are larger than this size range. This leads to a substantial decrease in $\kappa_{\mathrm{R}}$ per gram of dust in the disk midplane in these regions. Conversely, the high relative abundance of small grains at $z=H_{\text {gas }}$ (only small grains can be lifted to this height) leads to a strong enhancement in $\kappa_{\mathrm{R}}$ per gram of dust at this height. The $\kappa_{\mathrm{R}}$ profile at $z=R_{\mathrm{Hill}}$ and $z=\min \left(R_{\text {Hill }}, R_{\text {Bondi }}\right)$ in the top panel of Figure 9 can be understood using these same principles.

The dust-to-gas ratio increases with a higher concentration of large grains for a top-heavy size distribution, so we observe a flipped behavior for the $\epsilon$ ratio profile (middle panel of Figure 9), where it reaches lower values at higher altitudes beyond the ice line. Since larger grains settle close to the midplane, $\epsilon$ is highest at the midplane and decreases higher up. The $\epsilon$ evaluated at $\min \left(R_{\text {Hill }}, R_{\text {Bondi }}\right)$ converges to that of the midplane in the innermost and outermost regions. The former arises from efficient vertical mixing, whereas the latter materializes from $R_{\mathrm{Hill}} / H_{\text {gas }}$ and $R_{\mathrm{Bondi}} / H_{\text {gas }}$ approaching zero in the outer disk (see $R_{\mathrm{Hill}}$ and $R_{\text {Bondi }}$ profiles in Figure 7).

In the bottom panel of Figure 9, we plot the mean opacity per gram of protoplanetary disk material, which is the product of the quantities plotted in the top two panels. Regardless of our vertical location in the disk, we see the same precipitous decline in disk opacity as in the vertically integrated disk model. Notably, $\kappa_{\mathrm{R}}$ decreases by $\sim 2$ orders of magnitude between 0.1 and 5 au at the height of our fiducial planetary core's $R_{\text {Hill }}$. Within $\sim 10$ au, the $\kappa_{\mathrm{R}}$ profiles for the vertically integrated disk model and the different $z$ values are nearly identical. This happens within the ice line as a result of efficient vertical mixing of grains (i.e., both $\kappa_{\mathrm{R}}$ per gram of dust and $\epsilon$ are roughly constant as a function of $z$ ). Beyond the ice line and within $10 \mathrm{au}$, the sharp decline in $\epsilon$ with $z$ is counterbalanced by the increase in $\kappa_{\mathrm{R}}$ per gram of dust with $z$ to yield a weakly $z$-dependent $\kappa_{\mathrm{R}}$ (per gram of protoplanetary disk material).

\subsection{Time Evolution of the Dust Opacity}

Up to this point, we have presented results from our models after $1 \mathrm{Myr}$ of disk evolution. In this section, we explore the time-varying grain size distribution and dust-to-gas ratio from 0.1 to $10 \mathrm{Myr}$, where the lower limit is chosen to represent the plausible time at which massive planetary cores emerge. Figure 10 demonstrates that the absolute values of the dust-togas ratio and mean opacity throughout the disk tend to decline over time. This is due to the global depletion of dust in the disk as it gradually accretes onto the star. Because the timescale over which $\epsilon$ and $\kappa_{\mathrm{R}}$ evolve lengthens as time goes on, we present our results as a function of log time. Already by $0.1 \mathrm{Myr}$, the dust-to-gas ratio and $\kappa_{\mathrm{R}}$ profiles converge to shapes that are qualitatively similar to those of our fiducial $1 \mathrm{Myr}$ model. Although temporal evolution of the disk after 0.1 Myr leads to 1-2 orders of magnitude of decline in the dustto-gas ratio and opacity, it has a small effect on their radial gradient in the disk. However, there is a noticeable inward movement of the minima in the $\epsilon$ and $\kappa_{\mathrm{R}}$ profiles with time. This is because as the dust-to-gas ratio declines in the outer disk, the radius at which the disk transitions from being fragmentation-dominated to drift-dominated moves inward (Equation (16)). As we will show in Section 4, the overall decline in $\epsilon$ and $\kappa_{\mathrm{R}}$ over time leads to the enhancement of gas accretion onto planetary cores.

We note that the assumed disk size also plays an important role in the temporal evolution of the dust-to-gas ratio and, consequently, dust opacity. Due to radial drift, dust drains onto the star more rapidly in smaller disks, and the dust-to-gas ratio in the outer disk can become very small $\left(\lesssim 10^{-4}\right)$ as early as 1 Myr. Radial drift may be too efficient in disk models, and there is some tension with observations (e.g., Takeuchi \& 
Lin 2005; Brauer et al. 2008), as many disks with ages of a few megayears appear to have millimeter-sized grains present at large distances ( $\gtrsim 100$ s of au; e.g., Andrews et al. 2018; Hendler et al. 2020). Proposed solutions for resolving the radial drift problem include the presence of dust traps (e.g., Kretke \& Lin 2007; Pinilla et al. 2012; Zhu et al. 2014), larger-thanassumed disk gas density (Powell et al. 2019), and grains with large porosity (Estrada \& Cuzzi 2015; Garcia \& Gonzalez 2020). We circumvent this issue by modeling a relatively large and massive disk, ensuring a reasonable supply of dust throughout the disk lifetime.

\subsection{Temperature Structure of the Disk}

We have calculated the disk's temperature profile assuming a passively irradiated disk and thus assumed that it is identical for all of our grid models. However, accretional heating plays a role in setting the temperature structure of the disk, especially in the inner region and, in particular, for high disk viscosity (high $\alpha_{\mathrm{t}}$ ). In addition, dust dynamics and a variable $v_{\text {frag }}$ alter the dust-to-gas ratio and dust size distribution through the disk, leading to a location-dependent dust opacity. Ideally, our models would include a self-consistent coupling of the dust and gas dynamics with the disk temperature structure including the effect of heating due to accretion. Although this is beyond the scope of this work, we carry out a preliminary assessment of the effect that accretional heating and an enhanced dust-to-gas ratio would have on the temperature profile. In particular, we are interested in whether accretional heating has a significant impact on the location of the water-ice line, which marks the transition in $v_{\text {frag }}$ values.

We post-processed the results from our simulations to calculate the temperature structure of the disk using the method outlined in Birnstiel et al. (2010). The only difference in our method is that we self-consistently calculate the Rosseland and Planck mean opacities using the size distribution and dust-togas ratio from our simulations. To speed up the calculation of dust opacity, we use a power-law size distribution for the dust with a power-law index $\beta=-3.5$ and $a_{\max }$ set by our twopoppy simulations (see Section 3.2). We also account for the thermostat effect of dust vaporization at high temperatures (Birnstiel et al. 2010). When the temperature reaches $1500 \mathrm{~K}$, any further increase in the temperature leads to the vaporization of dust (and a decrease in opacity), so the temperature is kept stabilized at this value. Once the gas opacity alone (assumed to be $0.1 \mathrm{~cm}^{2} \mathrm{~g}^{-1}$ ) is enough to raise the temperature above $1500 \mathrm{~K}$, we assume that all of the dust has evaporated and allow the temperature to rise again.

We find that accounting for the effects of accretional heating and the elevated dust-to-gas ratio significantly increases the temperature of the disk inside $\sim 1$ au, but it has a negligible impact on the location of the water-ice line (Figure 11). For our fiducial model with $\alpha_{\mathrm{t}}=10^{-3}$, the ice-line location is essentially identical. For $\alpha_{\mathrm{t}}=10^{-4}$ and $10^{-2}$, the ice line moves from 0.75 to 0.4 and $1.6 \mathrm{au}$, respectively. We speculate that the increased temperature in the inner disk could also have an impact on the dust dynamics, as it would lead to a decrease in $\mathrm{St}_{\text {frag }}\left(\propto 1 / c_{\mathrm{s}}^{2}\right)$. This might further reduce the rate at which dust in the inner disk drains onto the star, thereby resulting in a larger dust pileup. This reinforces our conclusions about the difficulty of accreting gas in this region. However, obtaining a full solution to this problem would require us to couple the dust and gas dynamics with the disk temperature structure, which, to

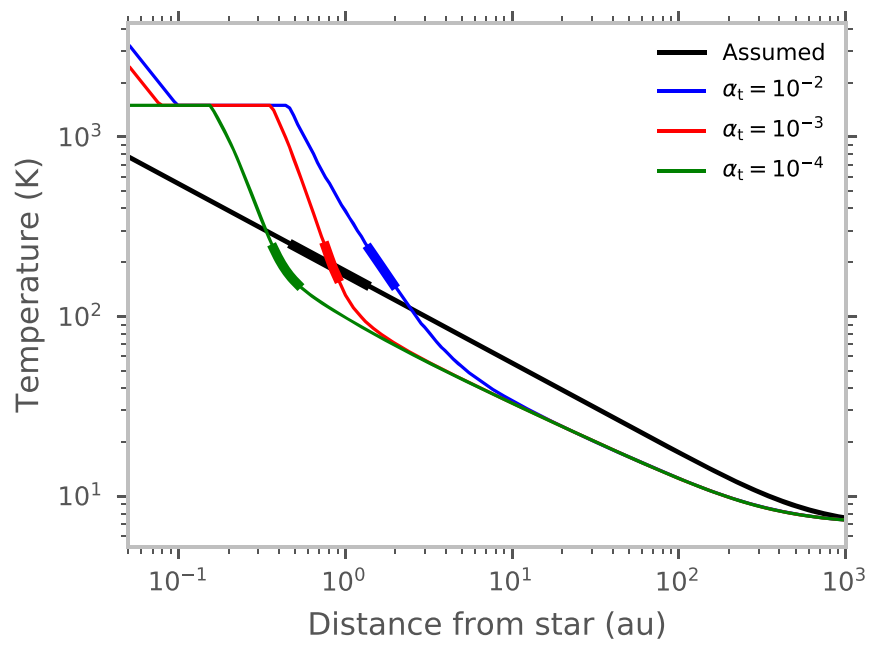

Figure 11. Post-processed temperature structure of the disk calculated using the method outlined in Birnstiel et al. (2010) with a self-consistent treatment of the opacity calculated using the size distribution and dust-to-gas ratio from our simulations. The disk properties at $t=1 \mathrm{Myr}$ are used to calculate the temperature structure and taken from simulations for which $v_{\text {frag }}$ is 1 and $10 \mathrm{~m} \mathrm{~s}^{-1}$ for ice-free and icy grains, respectively. The water-ice line $(150-250 \mathrm{~K})$ is marked with thick lines for each temperature profile.

the best of our knowledge, has only been attempted once in the published literature (Estrada et al. 2016).

\section{Implications for Planet Formation}

\subsection{Gas Accretion Mediated by Cooling}

Our calculated values for the dust opacity as a function of distance from the star show a dramatic decrease as we move beyond the ice line. We now consider what effect this variation in dust opacity and dust-to-gas ratio might have on the ability of planetary cores to accrete hydrogen-rich envelopes. For cores with masses $\lesssim 20 M_{\oplus}$, the rate of gas accretion onto the planetary core is initially regulated by the envelope's ability to cool and contract (e.g., Lee 2019). This cooling is controlled by the properties of the gas envelope at the innermost RCB, as most of the cooling luminosity is generated inside the innermost convective zone (Lee et al. 2014; Piso \& Youdin 2014).

There is a qualitative difference in the radiative-convective structure of planetary envelopes dominated by dust opacity versus gas opacity. For "dust-free" envelopes with negligible dust opacity, we expect to see a single convective zone that is connected to the disk via a nearly isothermal radiative zone. However, for "dusty" envelopes, where dust opacity dominates over gas opacity, the evaporation of dust grains deep inside the envelope leads to a dramatic drop in the local envelope opacity, which causes an intermediate radiative zone to form. Lee et al. (2014) showed that in this case, the innermost RCB appears at the $\mathrm{H}_{2}$ dissociation front $(\sim 2500 \mathrm{~K})$, where $\mathrm{H}^{-}$opacity starts to dominate.

We expect atmospheres to transition to the "dust-free" accretion regime when the dust opacity is comparable to the gas opacity at the relevant temperature. In the inner disk $(\sim 0.1 \mathrm{au})$, this transition occurs when the dust opacity approaches $\sim 0.01$ $\mathrm{cm}^{2} \mathrm{~g}^{-1}$. As we move farther out in the disk, the gas opacity decreases sharply $\left(\lesssim 10^{-4} \mathrm{~cm}^{2} \mathrm{~g}^{-1}\right.$ at the relevant densities; e.g., Freedman et al. 2014) as the number of available molecular line transitions decreases. In our fiducial disk model for a $15 M_{\oplus}$ core, the dust opacity at a height of $\min \left(R_{\mathrm{Hill}}\right.$, 
$R_{\text {Bondi }}$ ) above the midplane does not go below the gas opacity limit. Our models therefore predict that accretion at all orbital distance occurs in the "dusty" regime, whose RCB opacitywhich controls the rate of cooling and therefore accretion-is given by the $\mathrm{H}^{-}$opacity (Lee \& Chiang 2015):

$$
\begin{aligned}
\kappa\left(\mathrm{H}^{-}\right) \sim 3 & \times 10^{-2} \mathrm{~cm}^{2} \mathrm{~g}^{-1}\left(\frac{\rho}{10^{-4} \mathrm{~g} \mathrm{~cm}^{-3}}\right)^{0.5} \\
& \times\left(\frac{T}{2500 \mathrm{~K}}\right)^{7.5}\left(\frac{Z}{0.02}\right)^{1} .
\end{aligned}
$$

The only influence dust has on the $\mathrm{H}^{-}$opacity is via the metallicity-dependence $Z$ of the gas. We set $Z$ equal to the local dust-to-gas ratio in our gas accretion calculations, as the metals delivered via dust are present in the gas phase at the $\mathrm{H}_{2}$ dissociation front. Equating $Z$ to the dust-to-gas ratio is justified because the $Z$ dependence of $\kappa\left(\mathrm{H}^{-}\right)$results from its dependence on the availability of free electrons, most of which are sourced from metallic species. Although some of these metals might be present in the gas, the dust contribution dominates. This is likely to be true even in the most dustdepleted regions of the outer disk, as $\mathrm{CO}$ is predicted to be the dominant gas-phase metal in this region. This molecule does not dissociate until much deeper in the planetary atmosphere; hence, it will not contribute free electrons in the region where $\mathrm{H}^{-}$opacity becomes important.

We use this information to calculate gas accretion rates onto a planetary core as a function of disk location and time using the analytical scaling laws provided by Lee \& Chiang (2015), modified for the linear dependence on the bound radius and the weak dependence on the nebular density (see Lee \& Connors 2021). The GCR at time $t$ (with accretion beginning at $t_{0}$ ) in the "dusty" planetary envelope regime is given by

$$
\begin{aligned}
\mathrm{GCR} \sim & 0.06 f_{\mathrm{R}}\left(\frac{\Sigma_{g}}{2000 \mathrm{~g} \mathrm{~cm}^{-3}}\right)^{0.12}\left(\frac{t-t_{0}}{1 \mathrm{Myr}}\right)^{0.4}\left(\frac{\nabla_{\mathrm{ad}}}{0.17}\right)^{3.4} \\
& \times\left(\frac{2500 \mathrm{~K}}{T_{\mathrm{rcb}}}\right)^{4.8}\left(\frac{0.02}{Z}\right)^{0.4}\left(\frac{\mu_{\mathrm{rcb}}}{2.37}\right)^{3.4}\left(\frac{M_{\text {core }}}{5 M_{\oplus}}\right)^{1.7} .
\end{aligned}
$$

Here $f_{\mathrm{R}}$ is the bounded radius of a planet as a fraction of its $\min \left(R_{\text {Hill }}, R_{\text {Bondi }}\right)$, and we set it equal to 0.2 (e.g., Fung et al. 2019). The updated scaling law provided by Lee \& Connors (2021) also allows us to incorporate the dependence of the GCR on the gas surface density $\Sigma_{g}$, which we obtain from our disk model. The normalization factor of 0.06 is valid for $\Sigma_{g}<0.1 \times$ Minimum Mass Extrasolar Nebula $(\mathrm{MMEN})$ at $0.1 \mathrm{au}$. Here $\nabla_{\mathrm{ad}}, T_{\mathrm{RCB}}$, and $\mu_{\mathrm{RCB}}$ are the adiabatic gradient, temperature, and mean molecular weight evaluated at the RCB. We assume a fixed value of $T_{\mathrm{RCB}}=2500 \mathrm{~K}$ and $\nabla_{\mathrm{ad}}=0.17$, appropriate for the innermost $\mathrm{RCB}$ at the $\mathrm{H}_{2}$ dissociation front, for all of our calculations. We calculate $\mu_{\mathrm{RCB}}$ assuming $\mu=2.3$ for a pure hydrogen-helium mixture (solar abundance ratio) and $\mu=17$ for a pure metal-rich atmosphere. For the most metal-rich gases $(Z \gtrsim 0.2$; Lee \& Chiang 2016), the strong dependence of GCR on $\mu_{\mathrm{RCB}}$ dominates over the metallicitydependent increase in opacity, allowing for rapid accretion (Venturini et al. 2015). Our models predict that the dust-to-gas ratio throughout the disk will remain below this critical value for a majority of the disk lifetime. In the inner disk, $Z>0.2$ only at very early stages $(<0.1 \mathrm{Myr})$, when core formation is still likely ongoing. ${ }^{7}$ For the entirety of the duration of gas accretion that we model (0.1-10 Myr), an increased $Z$ therefore acts to reduce the accretion rate by increasing the gas opacity at the RCB. We incorporate the time dependence of $\Sigma_{g}, Z$, and $\mu_{\mathrm{RCB}}$ in our calculation of the GCR by numerically differentiating Equation (18) with respect to time and integrating between $t_{0}=0.1 \mathrm{Myr}$ (the emergence of the core) and the time $t$ (in the range $1-10 \mathrm{Myr}$ ) at which the planet stops accreting.

If a core reaches the threshold for runaway gas accretion $(\mathrm{GCR}=0.48$; Lee et al. 2014), we calculate the subsequent gas accretion rate using the minimum of the cooling-limited rate $\dot{M}_{\text {cool }}$, the disk accretion rate $\dot{M}_{\text {disk }}$, and the hydrodynamic accretion rate $\dot{M}_{\text {hydro }}$ (see Lee 2019 for an example):

$$
\begin{gathered}
\dot{M}_{\text {cool }}=0.48 \frac{M_{\text {core }}}{t_{\text {run }}} \exp \left(\frac{t}{t_{\text {run }}}\right), \\
\dot{M}_{\text {disk }}=3 \pi \nu \Sigma_{g}, \\
\dot{M}_{\text {hydro }}=0.29\left(\frac{M_{p}}{M_{*}}\right)^{4 / 3} \frac{\Sigma_{g}}{1+0.034 K}\left(\frac{r}{H_{\text {gas }}}\right)^{2} r^{2} \Omega_{K} .
\end{gathered}
$$

Here $t_{\text {run }}$ is the time taken by the core to reach a GCR $=0.48$ ( $t$ at which GCR $\left.=0.48-t_{0}\right), M_{p}=(\mathrm{GCR}+1) M_{\text {core }}$ is the total planet mass, and $K=\left(M_{p} / M_{*}\right)^{2} \alpha_{\mathrm{t}}^{-1}\left(H_{\mathrm{gas}} / r\right)^{-5}$ accounts for the depletion of gas surface density in the vicinity of the planet due to gap opening. This effect is only included in the gas accretion calculation and not in the evolution of the disk.

Figure 12 shows the GCR calculated for our fiducial core mass of $15 M_{\oplus}$ as a function of distance from the star at $t=1$ Myr. We vary the height $z_{\epsilon}$ from which material is accreted by the planet, which affects the metallicity (dust-to-gas ratio) of the accreted material and therefore the GCR profile. Along with our default value of $z_{\epsilon}=\min \left(R_{\mathrm{Hill}}, R_{\mathrm{Bondi}}\right)$, we also show GCR profiles for $z_{\epsilon}=[1 / 3,1,2] \times R_{\text {Hill }}$. Inside $\sim 1$ au, the relatively high $Z(\sim 0.1)$ produces a GCR in the range $0.06-0.08$ for a wide range of $z_{\epsilon}$. However, the sharp drop in $Z$ beyond $\sim 1$ au (see middle panel of Figure 9) leads to a rise in the amount of gas accreted by the planetary core, reaching a peak value of $\sim 0.2$ in the $1-10$ au region of the disk for $z_{\epsilon}=\min \left(R_{\text {Hill }}, R_{\text {Bondi }}\right)$. Beyond $\sim 10$ au, the metallicity of the gas (i.e., dust-to-gas ratio) at $R_{\mathrm{Hill}}$ and $R_{\mathrm{Bondi}}$ rises again as the Hill and Bondi radii shrink relative to the disk scale height, which leads to a decline in the GCR. The weak dependence of the GCR on $\Sigma_{g}$ also contributes to a decline in the GCR with distance. We note that the peak GCR value in the intermediate 1-10 au region increases with the height above the midplane from which the planet accretes, as the dust-to-gas ratio is a strongly decreasing function of $z$ in this region. Overall, Figure 12 demonstrates that the amount of gas accreted by a planetary core during the accretion-by-cooling phase, and hence its ability to reach the threshold for runaway growth, varies significantly as a function of its location in the disk.

\footnotetext{
7 We note that late-stage pollution of an envelope by ambient solids could enhance the interior metallicity beyond $Z \sim 0.2$ and trigger rapid gas accretion (Hori \& Ikoma 2011). The short dynamical timescale in the inner disk suggests that the solids there most likely lock into planetary cores before the late-stage disk gas dispersal, so such late-stage pollution is more likely to occur in the outer disk.
} 


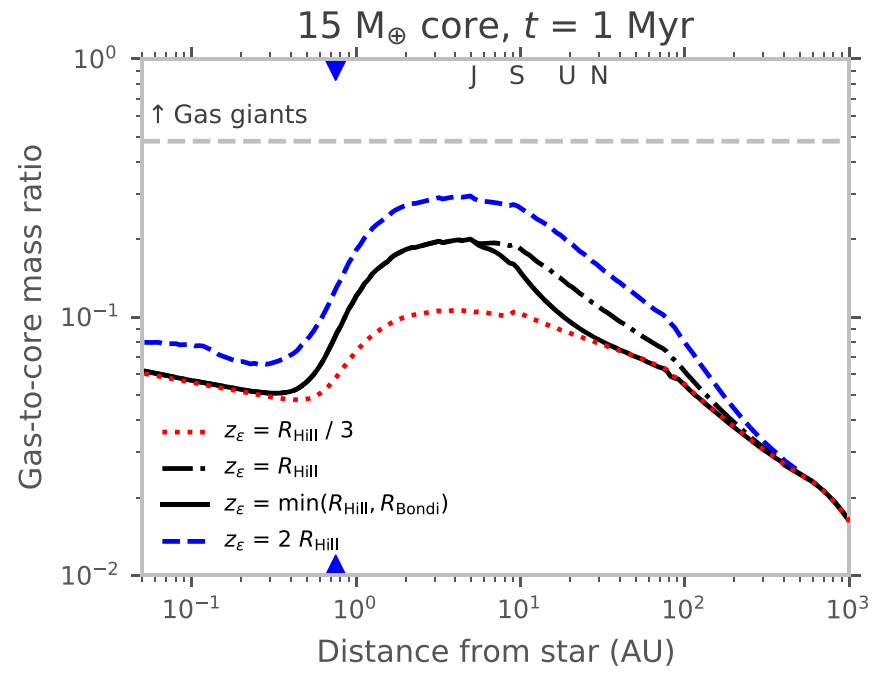

Figure 12. The GCR at $t=1 \mathrm{Myr}$ for a $15 M_{\oplus}$ core as a function of distance from the star, assuming the core starts accreting at $t_{0}=0.1 \mathrm{Myr}$. Here we vary the height $z_{\epsilon}$ from which gas is accreted by the planet. A GCR of 0.48 is marked with a dashed gray line, indicating the threshold for the onset of runaway gas accretion (Lee et al. 2014). The water-ice line is marked with blue triangles.

Figure 13 shows how the final GCR for a $15 M_{\oplus}$ core varies as a function of the assumed $\alpha_{\mathrm{t}}$ and $v_{\text {frag }}$ values. We calculate the GCR assuming that the core begins accreting at $t_{0}=1 \mathrm{Myr}$, as the dust-to-gas ratio at earlier times varies too rapidly for most of the models with $\alpha_{\mathrm{t}}=10^{-4}$ to allow us to use our simple gas accretion model. We also note that the high dust-togas ratio at $t \lesssim 1 \mathrm{Myr}$ and short orbital timescales in the inner disk for low $\nu_{\text {frag }}$ and $\alpha_{\mathrm{t}}=10^{-4}$ (Figure 5) would probably lead to efficient planetesimal formation, thereby reducing the dustto-gas ratio of the material that is available for gas accretion. The GCR calculations for these models at early times therefore require a more careful study of how the dust-to-gas ratio evolves if planetesimal formation occurs, which is beyond the scope of this work.

We find that for $\alpha_{\mathrm{t}}=10^{-2}$, the dust-to-gas ratio does not vary significantly with either location or time, and as a result, the GCR profiles lie in a relatively narrow range. The declining GCR with increasing distance is primarily due to the decline in gas surface density. In these high-viscosity models, a $15 M_{\oplus}$ core is unable to reach the threshold for runaway accretion anywhere in the disk. In contrast, models with $\alpha_{\mathrm{t}}=10^{-4}$ and $10^{-3}$ can produce a wide range of GCR values depending on the assumed values of $v_{\text {frag }}$. Models with substantial changes in $v_{\text {frag }}$ across the water-ice line result in a large radial variation in the GCR, with a sharp rise at $\sim 1$ au and a sharp fall at $\sim 10$ au, much more drastic than our fiducial model (Figures 12 and 14). These models are characterized by a deep minimum in the dustto-gas ratio at $\sim 1-10$ au that accelerates thermodynamic gas accretion, driving planets to runaway whose mass growth is eventually limited by the global disk accretion $\dot{M}_{\text {disk }}$ (when $\mathrm{GCR} \gtrsim 10$ ).

\subsection{Consequences for Giant Planet Formation and Demographics}

Our calculations provide a natural explanation for the observed peak in the gas-giant planet occurrence rate at $\sim 1-10$ au as measured by radial velocity and direct imaging surveys (e.g., Baron et al. 2019; Fernandes et al. 2019; Nielsen et al. 2019; Wittenmyer et al. 2020; Fulton et al. 2021). Figure 14 demonstrates that the location of the most favorable sites for rapid gas accretion is driven by the decrease in the dust-to-gas ratio just beyond the ice line, where relatively larger grains undergo efficient radial drift and vertical settling. We note that the nucleation of gas giants requires relatively massive cores $\left(\sim 15 M_{\oplus}\right)$ that assemble early (i.e., accrete gas for at least 3-10 Myr). Lighter cores and/or those that assemble late (i.e., accrete gas for a shorter amount of time) necessarily grow into planets with less massive envelopes. Although it is difficult to obtain good observational constraints on the core masses of extrasolar Jupiters (Thorngren \& Fortney 2019), we note that the cores of sub-Saturns-planets that were on the verge of runaway but were halted in growth before they became gas giants-are better constrained and appear to range between $\sim 15$ and $20 M_{\oplus}$ in the limiting case, where all metals are assumed to be sequestered in the core (Lopez \& Fortney 2014; Petigura et al. 2017). This range also agrees with core mass estimates derived from fitting mass-loss models to the sub-Saturn occurrence rate as a function of orbital period (Hallatt \& Lee 2021).

The same change in the fragmentation velocity of grains across the ice line that we invoke in our model may also result in the formation of more massive cores outside the ice line (e.g., Morbidelli et al. 2015; Venturini et al. 2020), reinforcing our results that gas giants are more likely to originate farther away from the star. Our work further demonstrates that the dust-to-gas ratio is expected to be radially variant and that it reaches a local minimum at a specific range of orbital distances (1-10 au), creating a preferred zone of rapid gas accretion. Qualitatively, our solar system also fits into our picture, with gas giants Jupiter and Saturn forming at intermediate distances, where the GCR peaks, and Uranus and Neptune forming further out, where the GCR declines with distance (Morbidelli et al. 2007; Batygin \& Brown 2010).

\subsection{Formation of Sub-Neptunes and Super-puffs}

Close-in sub-Neptunes appear to possess primordial hydrogen-rich envelopes that are a few percent of the total planet mass (e.g., Lopez \& Fortney 2014; Wolfgang \& Lopez 2015; Ning et al. 2018). Given their estimated core masses of 4-8 $M_{\oplus}$ (Wu 2019; Rogers \& Owen 2021), it is difficult to explain why these planets did not undergo runaway gas accretion and turn into gas giants, assuming that they formed in MMEN and accreted solar metallicity gas. Previous studies have proposed three potential solutions: (1) accretion of metal-rich gas, which increases the envelope opacity and slows the gas accretion rate during the cooling growth phase (e.g., Lee et al. 2014; Chen et al. 2020); (2) late-time core assembly, so that there is a very short period for the planet to accrete prior to the dispersal of the gas disk (Lee \& Chiang 2016); and (3) a flow of high-entropy gas into the Hill sphere of the growing planet that prevents it from cooling (Ormel et al. 2015; Béthune \& Rafikov 2019; but see Kurokawa \& Tanigawa 2018). Scenario 1 in and of itself applies for either dusty or dust-free accretion, but it is more effective for dusty accretion, as its overall higher opacity delays accretion even more. Our work revisits the first scenario in the context of in situ dusty gas accretion.

Our results suggest that the enhanced dust-to-gas ratio in the inner disk is sufficient to limit the envelope masses/accretion rates of sub-Neptunes forming in this region. We find that for a 

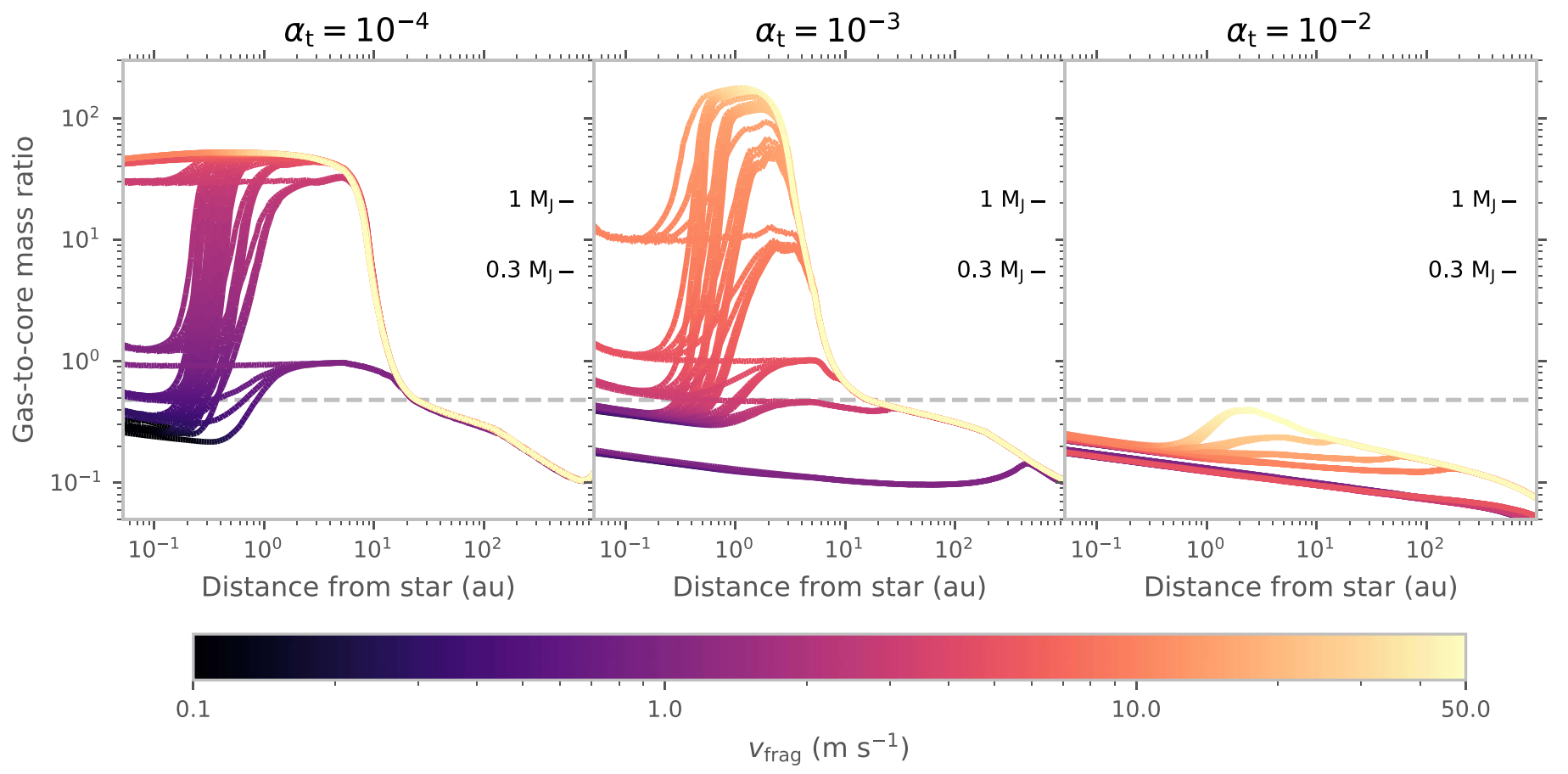

Figure 13. The GCR at $t=10 \mathrm{Myr}$ for a $15 M_{\oplus}$ core as a function of distance from the star, assuming the core starts accreting at $t_{0}=1 \mathrm{Myr}$, for our grid of models. We use a later $t_{0}$ here because the dust-to-gas ratio for the $\alpha_{\mathrm{t}}=10^{-4}$ model varies rapidly at earlier times, and our simple gas accretion model would therefore not be applicable. The dashed gray line indicates the threshold for the onset of runaway gas accretion (GCR of 0.48; Lee et al. 2014). The GCRs corresponding to planet masses of 0.3 and $1 M_{\mathrm{J}}$ are marked with black dashes; $0.3 M_{\mathrm{J}}$ is commonly used as a lower mass limit when calculating giant planet occurrence rates in radial velocity surveys (e.g., Cumming et al. 2008; Wittenmyer et al. 2020).

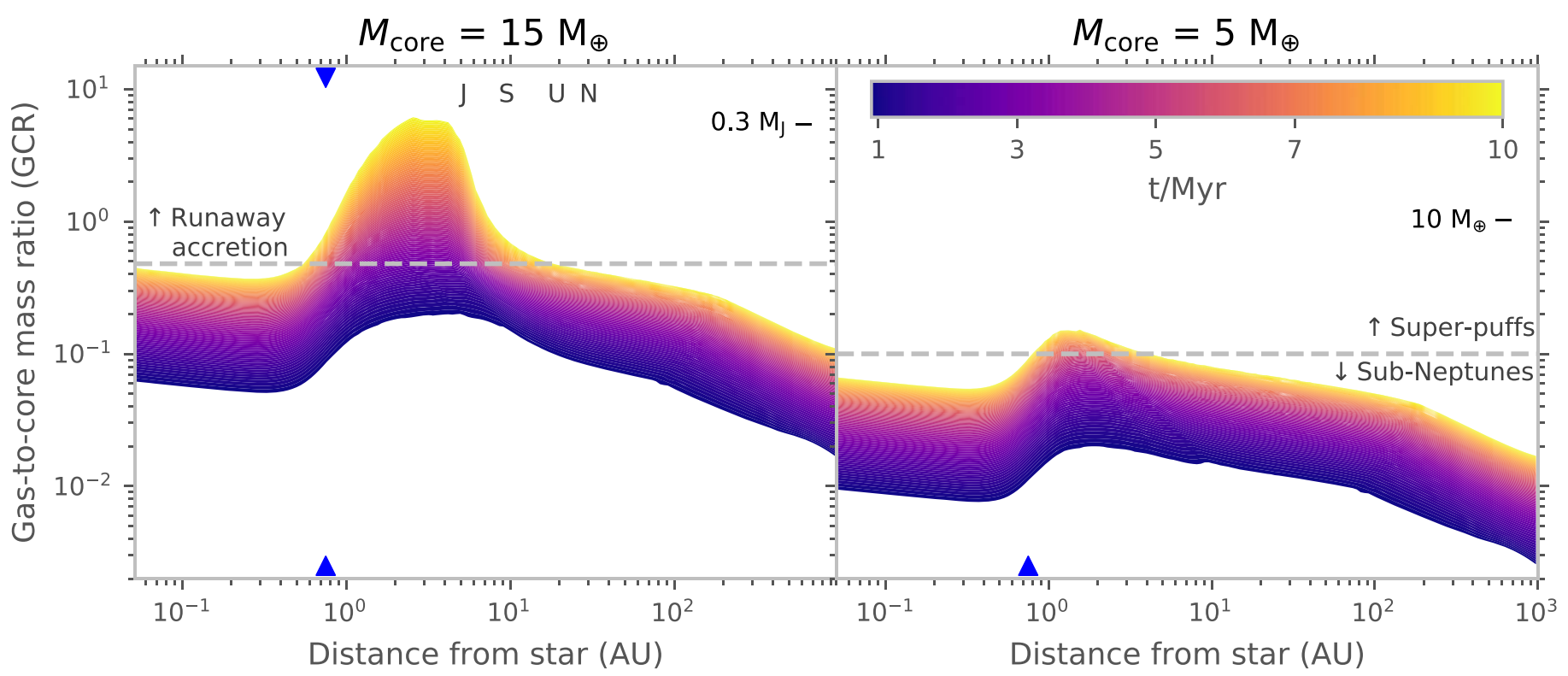

Figure 14. The GCR as a function of distance from the star for 15 (left panel) and 5 (right panel) $M_{\oplus}$ cores for time $t$ in the range $1-10$ Myr, assuming that they start accreting material present at $\min \left(R_{\mathrm{Hill}}, R_{\mathrm{Bondi}}\right)$ at $t_{0}=0.1 \mathrm{Myr}$. A GCR of 0.48 (onset of runaway gas accretion; Lee et al. 2014) and GCR $=0.1$ (for sub-Neptunes and super-puffs) are marked with dashed gray lines in the left and right panels, respectively. The GCRs corresponding to total planet masses of $0.3 M_{\mathbf{J}}\left(\right.$ left panel) and $10 M_{\oplus}$ (right panel) are indicated as well. The locations of solar system giant planets are marked along the abscissa at the top. The water-ice line is marked with blue triangles.

representative $5 M_{\oplus}$ core, the enhanced dust-to-gas ratio inside the ice line is enough to prevent the accretion of a massive gas envelope (Figure 14, right panel). If the metallicity is too high $(Z>0.2)$, the enhancement in the mean molecular weight of the gas can expedite gas accretion (Lee \& Chiang 2015; Venturini et al. 2015). For our fiducial choice of fragmentation velocities and turbulence parameter (as well as for a large swath of the parameter space), $Z$ stays below 0.2 in the inner disk after $0.1 \mathrm{Myr}$. As shown in Figure 14, a $5 M_{\oplus}$ core inside 1 au attains a few percent by mass envelope, consistent with the measured masses and radii of sub-Neptunes, even if the core assembled early and accreted gas for the full $10 \mathrm{Myr}$. We note that this result is not mutually exclusive with late-time core assembly for sub-Neptunes. The late-time, gas-poor environment favors the 
buildup of $\sim 5 M_{\oplus}$ sub-Neptune cores by a series of collisional mergers. Such mergers are necessary as the isolation masses, from either planetesimal (see Dawson \& Johnson 2018, their Figure 2) or pebble (see, e.g., Bitsch et al. 2018; Fung \& Lee 2018) accretion, are on the order of an Earth mass or smaller in the inner disk. Furthermore, late-time assembly of subNeptunes prevents inward migration of these planets once they assemble (Lee \& Chiang 2016).

Our models also provide support for previously published hypotheses about the origin of "super-puffs," a rare class of planets with giant planet-like radii (4-8 $R_{\oplus}$ ) and super-Earthlike masses (2-5 $M_{\oplus}$; Lee \& Chiang 2016). The low bulk densities of these planets imply that they possess hydrogen-rich envelopes that are tens of percent by mass (Jontof-Hutter et al. 2014; Masuda 2014; Ofir et al. 2014). Although the gas mass fraction of some super-puffs may be overestimated due to the inflation of planetary radii measurements by photochemical hazes lofted by outflowing gas, the majority of super-puffs do appear to have accreted substantially more gas than subNeptunes (Wang \& Dai 2019; Libby-Roberts et al. 2020; Gao \& Zhang 2020; Chachan et al. 2020). It is difficult to explain how these planets, which have core masses similar to those of sub-Neptunes, could have accreted an order of magnitude more gas in their present-day locations (Ikoma \& Hori 2012; Lee \& Chiang 2016). Lee \& Chiang (2016) proposed that super-puffs might form by accreting "dust-free" gas (dust opacity lower than gas opacity) beyond $\sim 1$ au. Although the dust opacity in our models is never low enough to qualify as dust-free, we find that this decrease in the dust-to-gas ratio beyond the ice line does indeed lead to significantly higher gas accretion rates and GCRs (Figure 14). All of the currently known super-puffs are in or near orbital resonances with other planets, ${ }^{8}$ which requires relatively smooth convergent migration (e.g., Cresswell \& Nelson 2006). This is consistent with a scenario in which super-puffs formed beyond $\sim 1$ au and then migrated inward via interactions with the protoplanetary gas disk. As Figure 14 shows, the creation of super-puffs requires their cores to have assembled early so that the total gas accretion time is longer. The requirement for early-stage core assembly is also in agreement with the migratory origin of super-puffs, as diskinduced migration requires a gas-rich environment.

\section{Discussion and Conclusions}

In this work, we use dust evolution models to demonstrate that the dust opacity and dust-to-gas ratio in protoplanetary disks are expected to be radially and vertically variant, with significant implications for planet formation. This is a result of grain growth and transport, which produce a highly nonuniform dust-to-gas ratio in the disk and generate top-heavy size distributions with grains that are orders of magnitude larger than the maximum grain size in the commonly assumed ISM distribution. We explore the sensitivity of our models to assumptions about the disk turbulence and fragmentation velocities and find that we obtain qualitatively similar results over a wide range of plausible values.

Models with a substantial difference in $v_{\text {frag }}$ across the ice line and moderate-to-low turbulence values $\alpha_{\mathrm{t}} \lesssim 10^{-3}$ produce the largest radial variations in the dust-to-gas ratio and dust

\footnotetext{
8 Most super-puffs orbit dim stars, which makes it hard to measure their masses with the radial velocity technique. Their masses have typically been determined by transit timing variations, which by definition require them to be in dynamically interacting multiplanet systems.
}

opacity. A large change in $v_{\text {frag }}$ across the ice line leads to a large difference in the Stokes number St of the largest grains within and beyond the ice line. In the inner disk with smaller St (well coupled to gas), dust grains pile up radially and mix well vertically. In the outer disk with larger St (more decoupled from gas), dust grains drift in rapidly and settle to the midplane. As a result, the inner disk is characterized by a high dust-to-gas ratio that is near constant with height, whereas the outer disk is characterized by a lower dust-to-gas ratio that decreases even further away from the midplane.

We use our location-dependent dust-to-gas ratio to calculate gas accretion rates onto planetary cores as a function of distance from the star. If we assume that the growing planet predominately accretes material present at $\min \left(R_{\mathrm{Hill}}, R_{\mathrm{Bondi}}\right)$ above the midplane, we find that the GCR is a strong function of its location in the disk. Within the ice line, gas accretion onto the core is suppressed by the high dust-to-gas ratio. At an intermediate distance beyond the ice line (1-10 au in our fiducial model), there is a steep decline in the dust-to-gas ratio, causing the GCR to rise and making it easier for cores to reach the threshold for runaway gas accretion. Beyond this point, the dust-to-gas ratio increases again as the growing planet accretes from a region closer to the disk midplane $\left(\min \left(R_{\text {Hill }}\right.\right.$, $\left.R_{\text {Bondi }}\right) / H_{\text {gas }}$ declines with distance). We conclude that dustgas dynamics favor gas-giant planet formation at intermediate distances, potentially explaining the peak in the giant planet occurrence rate versus orbital distance (e.g., Fulton et al. 2021). Our results also provide support for the hypothesis that superpuffs likely formed beyond the ice line, as the lower dust-to-gas ratio in this region can substantially accelerate their gas accretion rates.

We note that the same models presented in this study could be used to constrain the core mass distribution of gas-giant exoplanets by quantifying the fraction of planets that undergo runaway gas accretion as a function of location (e.g., Lee 2019). Previous studies of core formation have argued that a change in $v_{\text {frag }}$ across the ice line could lead to a significant increase in core masses outside the ice line (Morbidelli et al. 2015; Venturini et al. 2020). In a future study, we will explore whether the radially varying dust-to-gas ratio alone is sufficient to reproduce the observed mass-period distribution of gas-giant exoplanets, or whether it is also necessary to invoke a radially varying core mass function or large-scale migration. These same models could also be used to explore why outer gas giants are commonly accompanied by inner super-Earths (Zhu \& Wu 2018; Bryan et al. 2019).

In this study, we have limited ourselves to a single fiducial disk model to show how dust opacity varies with radial distance. However, observations of protoplanetary disks indicate that there is a large variation in disk properties, such as disk mass, size, lifetime, and metallicity, as well as the mass and luminosity of protostars (Andrews et al. 2018; Long et al. 2018, 2019). In future studies, we will investigate how dust evolution and gas accretion onto planetary cores depend on these properties and whether the diversity of exoplanets is thus linked to the diversity in disk and stellar properties.

Other potential improvements for these calculations include accounting for the conversion of dust to planetesimals/ planetary cores on the dust mass budget (likely to be important in the inner disk for $\alpha_{\mathrm{t}}=10^{-4}$ and low $v_{\text {frag }}$ ) and the effect of planet-disk interaction on dust growth and dynamics. In particular, as planetary cores become massive enough to 
perturb the gas disk, pressure maxima outside the planet's orbit trap some of the dust. This could affect the local size distribution and radial migration of dust, as well as the dustto-gas ratio of the material accreted by the growing planet (Chen et al. 2020). We expect these effects to be perturbative and more localized in nature and the global dust evolution to broadly follow the picture we have painted in this work. Overall, the radial variation of the dust-to-gas ratio and dust opacity has a substantial effect on the ability of planetary cores to accrete gas and should be considered in models of planet formation.

We are grateful to the referees for providing us with thoughtful suggestions that improved the paper. We are indebted to Dave Stevenson, Yanqin $\mathrm{Wu}$, and Chris Ormel for giving us feedback on this manuscript. Y.C. is grateful to Til Birnstiel for providing the excellent public repositories that this work relies on. Support for this work was provided by NASA through Space Telescope Science Institute grant GO15138.

\section{ORCID iDs}

Yayaati Chachan (1) https://orcid.org/0000-0003-1728-8269

Eve J. Lee (1) https://orcid.org/0000-0002-1228-9820

Heather A. Knutson (ib https://orcid.org/0000-0002-5375-4725

\section{References}

Akimkin, V., Vorobyov, E., Pavlyuchenkov, Y., \& Stoyanovskaya, O. 2020, MNRAS, 499, 5578

Alexander, D. R., \& Ferguson, J. W. 1994a, in Molecules in the Stellar Environment. Lecture Notes in Physics, ed. U. G. Jørgensen, Vol. 428 (Berlin: Springer), 149

Alexander, D. R., \& Ferguson, J. W. 1994b, ApJ, 437, 879

Andrews, S. M. 2015, PASP, 127, 961

Andrews, S. M., Huang, J., Pérez, L. M., et al. 2018, ApJL, 869, L41

Banzatti, A., Pinilla, P., Ricci, L., et al. 2015, ApJL, 815, L15

Baron, F., Lafrenière, D., Artigau, É, et al. 2019, AJ, 158, 187

Batalha, N. M., Rowe, J. F., Bryson, S. T., et al. 2013, ApJS, 204, 24

Batygin, K., \& Brown, M. E. 2010, ApJ, 716, 1323

Bell, K. R., \& Lin, D. N. C. 1994, Apj, 427, 987

Béthune, W., \& Rafikov, R. R. 2019, MNRAS, 488, 2365

Birnstiel, T., Andrews, S. M., Pinilla, P., \& Kama, M. 2015, ApJL, 813, L14

Birnstiel, T., Dullemond, C. P., \& Brauer, F. 2010, A\&A, 513, A79

Birnstiel, T., Dullemond, C. P., Zhu, Z., et al. 2018, ApJL, 869, L45

Birnstiel, T., Klahr, H., \& Ercolano, B. 2012, A\&A, 539, A148

Birnstiel, T., Ormel, C. W., \& Dullemond, C. P. 2011, A\&A, 525, 1

Bitsch, B., Johansen, A., Lambrechts, M., \& Morbidelli, A. 2015, A\&A, 575,28

Bitsch, B., Morbidelli, A., Johansen, A., et al. 2018, A\&A, 612, A30

Blum, J., \& Wurm, G. 2008, ARA\&A, 46, 21

Bowler, B. P., \& Nielsen, E. L. 2018, in Occurrence Rates from Direct Imaging Surveys, ed. H. J. Deeg \& J. A. Belmonte (Berlin: Springer), 155

Brauer, F., Dullemond, P., \& Henning, T. 2008, A\&A, 480, 859

Bryan, M. L., Knutson, H. A., Lee, E. J., et al. 2019, AJ, 157, 52

Carrera, D., Gorti, U., Johansen, A., \& Davies, M. B. 2017, ApJ, 839, 16

Chachan, Y., Jontof-Hutter, D., Knutson, H. A., et al. 2020, AJ, 160, 201

Chen, Y.-X., Li, Y.-P., Li, H., \& Lin, D. N. C. 2020, ApJ, 896, 135

Chiang, E., \& Youdin, A. 2010, AREPS, 38, 493

Chiang, E. I., \& Goldreich, P. 1997, ApJ, 490, 368

Cieza, L. A., Casassus, S., Tobin, J., et al. 2016, Natur, 535, 258

Cimerman, N. P., Kuiper, R., \& Ormel, C. W. 2017, MNRAS, 471, 4662

Cresswell, P., \& Nelson, R. P. 2006, A\&A, 450, 833

Cumming, A., Butler, R. P., Marcy, G. W., et al. 2008, PASP, 120, 531

D'Alessio, P., Calvet, N., \& Hartmann, L. 2001, ApJ, 553, 321

D’Alessio, P., Canto, J., Calvet, N., \& Lizano, S. 1998, ApJ, 500, 411

Dawson, R. I., \& Johnson, J. A. 2018, ARA\&A, 56, 175

Draine, B. T. 2003, ApJ, 598, 1026

Draine, B. T. 2006, ApJ, 636, 1114
Draine, B. T., \& Lee, H. M. 1984, ApJ, 285, 89

Drązkowska, J., \& Alibert, Y. 2017, A\&A, 608, 92

Dressing, C. D., \& Charbonneau, D. 2015, ApJ, 807, 45

Estrada, P. R., \& Cuzzi, J. N. 2015, AAS Meeting, 47, 507.02

Estrada, P. R., Cuzzi, J. N., \& Morgan, D. A. 2016, ApJ, 818, 200

Fernandes, R. B., Mulders, G. D., Pascucci, I., Mordasini, C., \& Emsenhuber, A. 2019, ApJ, 874, 81

Flaherty, K. M., Hughes, A. M., Rose, S. C., et al. 2017, ApJ, 843, 150

Flaherty, K. M., Hughes, A. M., Rosenfeld, K. A., et al. 2015, ApJ, 813, 99

Flaherty, K. M., Hughes, A. M., Teague, R., et al. 2018, ApJ, 856, 117

Freedman, R. S., Lustig-Yaeger, J., Fortney, J. J., et al. 2014, ApJS, 214, 25

Fressin, F., Torres, G., Charbonneau, D., et al. 2013, ApJ, 766, 81

Fromang, S., \& Nelson, R. P. 2009, A\&A, 496, 597

Fulton, B. J., Rosenthal, L. J., Hirsch, L. A., et al. 2021, ApJS, 255, 14

Fung, J., Artymowicz, P., \& Wu, Y. 2015, ApJ, 811, 101

Fung, J., \& Lee, E. J. 2018, ApJ, 859, 126

Fung, J., Zhu, Z., \& Chiang, E. 2019, ApJ, 887, 152

Gao, P., \& Zhang, X. 2020, ApJ, 890, 93

Garcia, A. J. L., \& Gonzalez, J.-F. 2020, MNRAS, 493, 1788

Gundlach, B., \& Blum, J. 2015, ApJ, 798, 34

Gundlach, B., Schmidt, K. P., Kreuzig, C., et al. 2018, MNRAS, 479, 1273

Hallatt, T., \& Lee, E. J. 2021, arXiv:2105.12746

Hayashi, C. 1981, PThPS, 70, 35

Hendler, N., Pascucci, I., Pinilla, P., et al. 2020, ApJ, 895, 126

Henning, T., \& Stognienko, R. 1996, A\&A, 311, 303

Hori, Y., \& Ikoma, M. 2011, MNRAS, 416, 1419

Howard, A. W., Marcy, G. W., Bryson, S. T., et al. 2012, ApJS, 201, 15

Ikoma, M., \& Hori, Y. 2012, ApJ, 753, 66

Ikoma, M., Nakazawa, K., \& Emori, H. 2000, ApJ, 537, 1013

Jontof-Hutter, D., Lissauer, J. J., Rowe, J. F., \& Fabrycky, D. C. 2014, ApJ, 785,15

Kimura, H., Wada, K., Kobayashi, H., et al. 2020, MNRAS, 1813, 1801

Kitzmann, D., \& Heng, K. 2018, MNRAS, 475, 94

Kretke, K. A., \& Lin, D. N. C. 2007, ApJL, 664, L55

Kurokawa, H., \& Tanigawa, T. 2018, MNRAS, 479, 635

Lambrechts, M., \& Lega, E. 2017, A\&A, 606, A146

Laor, A., \& Draine, B. T. 1993, ApJ, 402, 441

Lee, E. J. 2019, ApJ, 878, 36

Lee, E. J., \& Chiang, E. 2015, ApJ, 811, 41

Lee, E. J., \& Chiang, E. 2016, ApJ, 817, 90

Lee, E. J., Chiang, E., \& Ormel, C. W. 2014, ApJ, 797, 95

Lee, E. J., \& Connors, N. J. 2021, ApJ, 908, 32

Libby-Roberts, J. E., Berta-Thompson, Z. K., Désert, J.-M., et al. 2020, AJ, 159,57

Long, F., Herczeg, G. J., Harsono, D., et al. 2019, ApJ, 882, 49

Long, F., Pinilla, P., Herczeg, G. J., et al. 2018, ApJ, 869, 17

Lopez, E. D., \& Fortney, J. J. 2014, ApJ, 792, 1

Lynden-Bell, D., \& Pringle, J. E. 1974, MNRAS, 168, 603

Masuda, K. 2014, ApJ, 783, 53

Mathis, J. S., Rumpl, W., \& Nordsieck, K. H. 1977, ApJ, 217, 425

Miyake, K., \& Nakagawa, Y. 1993, Icar, 106, 20

Morbidelli, A., Lambrechts, M., Jacobson, S., \& Bitsch, B. 2015, Icar, 258, 418

Morbidelli, A., Tsiganis, K., Crida, A., Levison, H. F., \& Gomes, R. 2007, AJ, 134,1790

Mordasini, C. 2018, Handbook of Exoplanets (Cham: Springer International Publishing), 2425

Morton, T. D., \& Swift, J. 2014, ApJ, 791, 10

Mulders, G. D., \& Dominik, C. 2012, A\&A, 539, 9

Musiolik, G., \& Wurm, G. 2019, ApJ, 873, 58

Nielsen, E. L., De Rosa, R. J., Macintosh, B., et al. 2019, AJ, 158, 13

Ning, B., Wolfgang, A., \& Ghosh, S. 2018, ApJ, 869, 5

Oberg, K. I., \& Bergin, E. A. 2021, PhR, 893, 1

Oberg, K. I., Murray-Clay, R., Bergin, E. A., et al. 2011, ApJL, 743, L16

Ofir, A., Dreizler, S., Zechmeister, M., \& Husser, T.-O. 2014, A\&A, 561, A103

Ormel, C. W., \& Cuzzi, J. N. 2007, A\&A, 466, 413

Ormel, C. W., Shi, J.-M., \& Kuiper, R. 2015, MNRAS, 447, 3512

Petigura, E. A., Marcy, G. W., Winn, J. N., et al. 2018, AJ, 155, 89

Petigura, E. A., Sinukoff, E., Lopez, E. D., et al. 2017, AJ, 153, 142

Pinilla, P., Birnstiel, T., Ricci, L., et al. 2012, A\&A, 538, A114

Pinilla, P., Klarmann, L., Birnstiel, T., et al. 2016, A\&A, 585, A35

Pinilla, P., Pohl, A., Stammler, S. M., et al. 2017, ApJ, 845, 68

Pinte, C., Dent, W. R. F., Menard, F., et al. 2016, ApJ, 816, 25

Piso, A. M. A., \& Youdin, A. N. 2014, ApJ, 786, 21

Piso, A.-M. A., Youdin, A. N., Murray-Clay, R. A., et al. 2015, ApJ, 800, 82 Pollack, J. B., Hubickyj, O., Bodenheimer, P. H., et al. 1996, Icar, 124, 62 
Poppe, T., Blum, J., \& Henning, T. 2000, ApJ, 533, 454

Powell, D., Murray-Clay, R., Pérez, L. M., Schlichting, H. E., \& Rosenthal, M. 2019, ApJ, 878, 116

Rogers, J. G., \& Owen, J. E. 2021, MNRAS, 503, 1526

Rosenthal, L. J., Fulton, B. J., Hirsch, L. A., et al. 2021, ApJS, 255, 8

Rosenthal, M. M., \& Murray-Clay, R. A. 2018, ApJ, 864, 66

Savvidou, S., Bitsch, B., \& Lambrechts, M. 2020, A\&A, 640, 63

Shakura, N. I., \& Sunyaev, R. A. 1973, A\&A, 24, 337

Steinpilz, T., Teiser, J., \& Wurm, G. 2019, ApJ, 874, 60

Stevenson, D. J. 1982, P\&SS, 30, 755

Takeuchi, T., \& Lin, D. N. C. 2005, ApJ, 623, 482

Testi, L., Natta, A., Shepherd, D. S., \& Wilner, D. J. 2003, A\&A, 403, 323

Thorngren, D., \& Fortney, J. J. 2019, ApJL, 874, L31

Turner, N. J., Fromang, S., Gammie, C., et al. 2014, in Protostars and Planets VI, ed. H. Beuther et al. (Tuscon, AZ: Univ. of Arizona Press), 411

van de Hulst, H. C. 1957, Light Scattering by Small Particles (New York: John Wiley \& Sons)
Venturini, J., Alibert, Y., Benz, W., \& Ikoma, M. 2015, A\&A, 576, A114

Venturini, J., Guilera, O. M., Ronco, M. P., \& Mordasini, C. 2020, A\&A, 644, A174

Wada, K., Tanaka, H., Okuzumi, S., et al. 2013, A\&A, 559, A62

Wang, L., \& Dai, F. 2019, ApJL, 873, L1

Warren, S. G., \& Brandt, R. E. 2008, JGRA, 113, D14220

Weidenschilling, S. 1977, MNRAS, 180, 57

Wittenmyer, R. A., Butler, R. P., Tinney, C. G., et al. 2016, ApJ, 819, 28

Wittenmyer, R. A., Wang, S., Horner, J., et al. 2020, MNRAS, 492, 377

Wolfgang, A., \& Lopez, E. 2015, ApJ, 806, 183

Wu, Y. 2019, ApJ, 874, 91

Zhang, M., Chachan, Y., Kempton, E. M.-R., \& Knutson, H. A. 2019, PASP, 131,034501

Zhang, M., Chachan, Y., Kempton, E. M.-R., Knutson, H. A., \& Chang, W. H. 2020, ApJ, 899, 27

Zhu, W., \& Wu, Y. 2018, AJ, 156, 92

Zhu, Z., Stone, J. M., Rafikov, R. R., \& Bai, X.-n. 2014, ApJ, 785, 122 